\title{
Mimicking Nonequilibrium Steady States with Time-Periodic Driving
}

\author{
O. Raz, ${ }^{1, *}$ Y. Subaş1, ${ }^{1}$ and C. Jarzynski ${ }^{1,2}$ \\ ${ }^{1}$ Department of Chemistry and Biochemistry, University of Maryland, \\ College Park, Maryland 20742, USA \\ ${ }^{2}$ Institute for Physical Science and Technology, University of Maryland, \\ College Park, Maryland 20742, USA
}

(Received 30 October 2015; revised manuscript received 7 April 2016; published 18 May 2016)

\begin{abstract}
Under static conditions, a system satisfying detailed balance generically relaxes to an equilibrium state in which there are no currents. To generate persistent currents, either detailed balance must be broken or the system must be driven in a time-dependent manner. A stationary system that violates detailed balance evolves to a nonequilibrium steady state (NESS) characterized by fixed currents. Conversely, a system that satisfies instantaneous detailed balance but is driven by the time-periodic variation of external parametersalso known as a stochastic pump (SP)—reaches a periodic state with nonvanishing currents. In both cases, these currents are maintained at the cost of entropy production. Are these two paradigmatic scenarios effectively equivalent? For discrete-state systems, we establish a mapping between nonequilibrium stationary states and stochastic pumps. Given a NESS characterized by a particular set of stationary probabilities, currents, and entropy production rates, we show how to construct a SP with exactly the same (time-averaged) values. The mapping works in the opposite direction as well. These results establish a proof of principle: They show that stochastic pumps are able to mimic the behavior of nonequilibrium steady states, and vice versa, within the theoretical framework of discrete-state stochastic thermodynamics. Nonequilibrium steady states and stochastic pumps are often used to model, respectively, biomolecular motors driven by chemical reactions and artificial molecular machines steered by the variation of external, macroscopic parameters. Our results loosely suggest that anything a biomolecular machine can do, an artificial molecular machine can do equally well. We illustrate this principle by showing that kinetic proofreading, a NESS mechanism that explains the low error rates in biochemical reactions, can be effectively mimicked by a constrained periodic driving.
\end{abstract}

DOI: 10.1103/PhysRevX.6.021022

Subject Areas: Statistical Physics

\section{INTRODUCTION AND MOTIVATION}

While there is currently no single theory that unifies all nonequilibrium phenomena, a number of useful paradigms of nonequilibrium behavior have emerged. These include small perturbations near equilibrium [1], systems driven away from an initial state of equilibrium [2], spontaneous relaxation towards equilibrium [3], nonequilibrium steady states generated by fixed thermodynamic forces [4], and stochastic pumps driven by the time-periodic variation of external parameters [5,6]. Theoretical frameworks developed within each paradigm-for instance, linear response theory to describe near-equilibrium perturbations [7] - have contributed to a broader understanding of nonequilibrium processes.

\footnotetext{
* Corresponding author. orenraz@gmail.com

Published by the American Physical Society under the terms of the Creative Commons Attribution 3.0 License. Further distribution of this work must maintain attribution to the author(s) and the published article's title, journal citation, and DOI.
}

In this work, we focus on two of these paradigms: nonequilibrium steady states and stochastic pumps. These share certain features, notably the persistence of nonvanishing currents and entropy production, which invite a comparison between the two. As elaborated below, we devise a mapping from one paradigm to the other; given a system in a nonequilibrium steady state characterized by certain occupation probabilities, currents, and entropy production rates, we show how to construct a stochastic pump that exhibits the same (time-averaged) properties. The inverse direction, namely, the construction of a nonequilibrium steady state with the same properties as a given, time-averaged stochastic pump, will also be discussed.

In the nonequilibrium steady-state (NESS) paradigm, a system driven by fixed thermodynamic forces-such as temperature gradients or chemical potential differencesreaches a steady state in which its statistical properties are stationary with time. Unlike an equilibrium state, a nonequilibrium steady state exhibits nonvanishing currents, reflecting the violation of detailed balance. In order to maintain such a state, a thermodynamic cost must be paid. This cost is measured by the continual depletion of a 
thermodynamic resource, such as a chemical fuel, resulting in the production of entropy in the system's thermal surroundings.

Biomolecular motors illustrate the NESS paradigm $[8,9]$ : A reaction such as $A T P$ hydrolysis $\left(A T P \rightarrow A D P+P_{i}\right)$ produces entropy in the surrounding solution, and the chemical potential difference between reactants and products provides the thermodynamic force. The "current" in this situation corresponds to the mechanical motion produced by the motor, for instance, the stepping motion of the kinesin motor toward the positive end of a microtubule filament $[10,11]$, or the rotary motion of the $\mathrm{F} 1$ domain in $A T P$ synthase $[12,13]$. For a recent review of the stochastic theory of nonequilibrium steady states, as applied to biochemical processes, see Ref. [4].

In the stochastic pump paradigm, a system is driven by the time-periodic variation of external parameters in the presence of a thermal reservoir. It is typically assumed that the dynamics satisfy detailed balance at every instant in timein other words, if the parameters were suddenly frozen at their instantaneous values, the system would relax to equilibrium. Under suitable conditions, a periodically driven system reaches a time-periodic state with nonvanishing time-averaged currents. These currents are effectively "pumped" by the periodic variation of the parameters, and the cost associated with pumping these currents is the work invested in driving the parameters. Ultimately, the energy provided by this work is dissipated into the thermal reservoir, resulting in the production of entropy.

The study of stochastic pumps has been stimulated by experiments on artificial molecular machines [14-16], which are manipulated by the variation of external parameters to achieve some desired behavior. For instance, in experiments on catenanes-mechanically interlocked ringlike molecules - the aim was to produce unidirectional rotation of one ring around the other [17]. Theoretical investigations of SP have focused on slowly driven $[5,18]$ as well as weakly driven [19] pumps, "no-pumping" theorems [20-24], the role of interactions [25] and fluctuations [26], and the ability to extract work from stochastic pumps [27].

Underlying both the experimental work on artificial molecular machines and the theoretical work on stochastic pumps is the broad goal of understanding how to achieve controlled, directed motion at the molecular level. The focus on time-dependent driving is motivated in part by the difficulty of synthesizing artificial molecular systems that take advantage of chemical potential differences to generate motion. It is often simpler to manipulate the system by varying external parameters such as temperature and the surrounding chemical environment. From a theoretical perspective, it is then natural to investigate the range of behaviors that can be achieved when driving a stochastic system by varying its parameters. Biomolecular motors driven by chemical reactions are capable of accomplishing a remarkably diverse array of tasks. In principle, are artificial molecular machines driven by time-periodic forcing equally versatile?

In both nonequilibrium steady states and stochastic pumps, the generated currents can be viewed as desired outcomes, and entropy production is the cost of achieving them. In this perspective, fixed thermodynamic forces (NESS) and time-periodic external driving (stochastic pump, SP) represent the tools at our disposal. We compare these two sets of tools with respect to the degree of control that can be achieved. In particular, we investigate whether time-periodic driving can always produce the same outcome (identical currents) as fixed thermodynamic forces, and at the same cost (identical entropy production), after time averaging. In other words, can a stochastic pump "mimic" an arbitrary nonequilibrium steady state?

Somewhat different aspects of the relationship between nonequilibrium steady states and stochastic pumps have recently been addressed by two other groups. Esposito and Parrondo [28] have shown that hidden, rapidly driven, reversible stochastic pumps can generate currents that- to an observer who is unaware of the pumps-appear to violate detailed balance. Uzdin, Levy, and Kosloff [29] have established a thermodynamic equivalence between three types of quantum heat engines, in the limit of weak thermalization and a weak driving field.

A different context in which similar ideas were recently suggested is topological insulators [30,31], where temporal periodic driving can be used to mimic material properties that are difficult to achieve in equilibrium. In these systems, commonly denoted as "Floquet topological insulators," the properties of interest are not the probabilities, currents, and entropy production rates as in our case, but rather the topological classification of the effective Hamiltonian and the existence of protected edge states. In recent years, Floquet topological insulators have been experimentally demonstrated in various setups $[32,33]$.

In this manuscript, we begin by considering a generic Markov process of random transitions among $n$ states of a system. The transition rates are fixed in time and do not satisfy detailed balance; hence, the dynamics lead to a NESS with nonvanishing currents and entropy production. We then show how to prescribe a stochastic pump that has, in the limit of many cycles, the same time-averaged probabilities, currents, and entropy production rates as the NESS. This prescription is constructive though not unique. Surprisingly, the construction does not require the solution of any differential equations, only linear algebraic equations. By contrast, a mapping in the opposite direction (from SP to NESS) requires that we first determine the periodic state of the system, which involves solving a set of coupled ordinary differential equations with time-periodic parameters. Typically, this can only be done numerically.

In some applications, transition rates are constrained by experimental or fundamental considerations. An example 
for fundamentally constrained rates is kinetic proofreading $[34,35]$, where the ratio between the steady-state probabilities of two specific states is controlled without manipulating their energies. Kinetic proofreading provides a model for understanding how low error rates in biomolecular synthesis can be achieved at the cost of hydrolyzing ATP into $A D P+P_{i}$. As an application for the tools developed in this manuscript, it is shown that kinetic proofreading in a three-state system can be achieved by SP, with exactly the same controllability over the time-averaged probability distribution and at exactly the same cost in terms of entropy production rates, as in the NESS kinetic proofreading.

The rest of the manuscript is organized as follows: In Sec. IV, we formulate the problem and review some useful known results. We then consider in Sec. V the simpler direction, namely, mapping a stochastic pump into a NESS. In Sec. VI, we present two types of transformations for detailed balance matrices, which play a key role in our construction of a SP that mimics NESS, and the "no-current-loops" property that sets a constraint on pumping with a time-dependent detailed balance matrix. The construction of a pumping protocol is described in Sec. VII. As an application, NESS and SP kinetic proofreading of a three-state system are analyzed in Sec. VI. We finish with a few concluding remarks in Sec. VII.

\section{SETUP AND BACKGROUND}

\section{A. Definitions}

We consider an ergodic, continuous-time Markovian system with $n$ states. The evolution of the system consists of random, Poissonian transitions among these states, with transition rates that are governed either by a timeindependent rate matrix $\mathcal{R}$ (when analyzing nonequilibrium steady states) or a time-periodic rate matrix $\mathcal{W}(t)$ (for stochastic pumps), as described in more detail below. It is convenient to picture the system in terms of a "connectivity graph" with $n$ nodes and a finite number of edges connecting

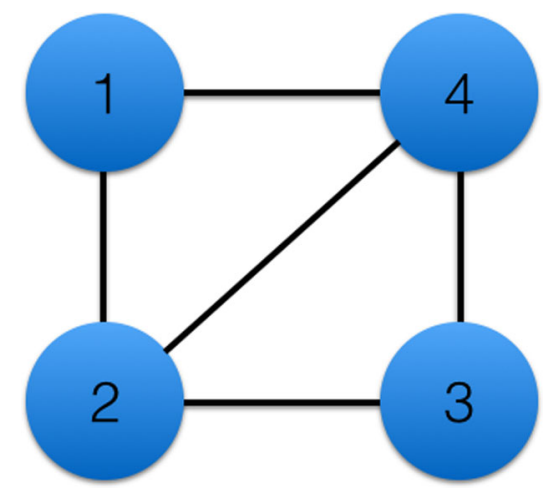

FIG. 1. A four-state system described by a graph. Each node represents a state of the system. The edges represent nonvanishing transition rates between states. In this example, direct transitions between states 1 and 3 are not allowed. given pairs of nodes. An edge between states $i$ and $j$ implies that the system can make transitions between these states (see Fig. 1). We use $\vec{p}(t)$ to denote the vector of the probabilities whose $i$ th component $p_{i}(t)$ is the probability for the system to be in state $i$ at time $t$.

In the NESS scenario, the evolution of the system obeys the master equation

$$
\partial_{t} \vec{p}=\mathcal{R} \vec{p} .
$$

For $i \neq j$, the matrix element $\mathcal{R}_{i j}$ is the probability per unit time for a system in state $j$ to make a transition to state $i$. The diagonal elements of $\mathcal{R}$ are negative and are determined by conservation of probability: $\sum_{i} \mathcal{R}_{i j}=0$. For the system to be ergodic, we demand that (i) if $\mathcal{R}_{i j} \neq 0$, then also $\mathcal{R}_{j i} \neq 0$, and (ii) the graph associated with $\mathcal{R}$ is connected. In other words, for any pair of nodes (states) $j$ and $i$, there exists a path from $j$ to $i$, possibly through a sequence of intermediate nodes, along the edges of the graph. Under these conditions, Eq. (1) has a unique steadystate solution, which we denote by $\vec{p}^{s s}$, and any solution of Eq. (1) converges to this steady state in the long-time limit [36].

In addition to the steady-state probabilities $\vec{p}^{s s}$, we are interested in the steady-state currents, defined by

$$
\mathcal{J}_{i j}^{s s}=\mathcal{R}_{i j} p_{j}^{s s}-\mathcal{R}_{j i} p_{i}^{s s},
$$

and the entropy production rates associated with these currents [36,37]:

$$
\sigma_{i j}^{s s}=\mathcal{J}_{i j}^{s s} \log \frac{\mathcal{R}_{i j} p_{j}^{s s}}{\mathcal{R}_{j i} p_{i}^{s s}} .
$$

We assume that some of these currents, and therefore the corresponding entropy production rates, are nonvanishing. In other words, we assume the dynamics violate detailed balance; hence, $\vec{p}^{s s}$ is a genuinely nonequilibrium steady state.

In the stochastic pump scenario, the system obeys a master equation with a time-periodic rate matrix,

$$
\partial_{t} \vec{p}=\mathcal{W}(t) \vec{p},
$$

where

$$
\mathcal{W}(t)=\mathcal{W}(t+T)
$$

for some finite period $T$. If we momentarily treat $t$ in Eq. (4) as a parameter of the rate matrix (rather than as the time variable), then for any fixed value of this parameter, we assume the rate matrix $\mathcal{W}(t)$ has a unique stationary solution $\vec{\pi}(t)$, and we further assume that

$$
\mathcal{W}_{i j}(t) \pi_{j}(t)-\mathcal{W}_{j i}(t) \pi_{i}(t)=0 \quad \forall i, j, t .
$$

In other words, the dynamics generated by $\mathcal{W}(t)$ (for fixed $t$ ) satisfy detailed balance. We refer to $\vec{\pi}(t)$ as the 
equilibrium state of $\mathcal{W}(t)$. This is the state to which the system would relax if all the rates $\mathcal{W}_{i j}$ were "frozen" in time. Note that in our notation, $\mathcal{R}_{i j}$ denotes a nondetailedbalance rate matrix, and $\mathcal{W}_{i j}$ represent a detailed-balance rate matrix. Therefore, NESS systems are associated with a rate matrix $\mathcal{R}_{i j}$, whereas SP systems are described through a periodic $\mathcal{W}_{i j}(t)$, though in Sec. VI, we also refer to a time-independent detailed-balance rate matrix as $\mathcal{W}_{i j}$.

Let us now return to thinking of $t$ as time. For any solution of Eq. (4), the quantities

$$
\begin{gathered}
\mathcal{J}_{i j}(t)=\mathcal{W}_{i j} p_{j}-\mathcal{W}_{j i} p_{i}, \\
\sigma_{i j}(t)=\mathcal{J}_{i j} \log \frac{\mathcal{W}_{i j} p_{j}}{\mathcal{W}_{j i} p_{i}}
\end{gathered}
$$

(suppressing the argument $t$ on the right side) represent instantaneous currents and entropy production rates. Under Eqs. (4) and (5), the statistical state of the system evolves asymptotically to a unique time-periodic state,

$$
\vec{p}^{p s}(t)=\vec{p}^{p s}(t+T),
$$

with currents and entropy production rates

$$
\begin{aligned}
\mathcal{J}_{i j}^{p s}(t) & =\mathcal{W}_{i j} p_{j}^{p s}-\mathcal{W}_{j i} p_{i}^{p s}, \\
\sigma_{i j}^{p s}(t) & =\mathcal{J}_{i j}^{p s} \log \frac{\mathcal{W}_{i j} p_{j}^{p s}}{\mathcal{W}_{j i} p_{i}^{p s}} .
\end{aligned}
$$

These are analogous to the quantities appearing in Eqs. (2) and (3), only $\mathcal{J}_{i j}^{p s}(t)$ and $\sigma_{i j}^{p s}(t)$ are periodic with time, whereas $\mathcal{J}_{i j}^{s s}$ and $\sigma_{i j}^{s s}$ do not vary with time.

Throughout this paper, we are interested in the asymptotic properties of the system; therefore, we consider only the steady state $\vec{p}^{s s}$ and the periodic state $\vec{p}^{p s}(t)$, and not the process of relaxation to either of these states.

In order to compare the NESS and stochastic pump (SP) scenarios, let us define the time-averaged quantities in the periodic state of the SP:

$$
\begin{aligned}
& \overline{p_{i}^{p s}}=\frac{1}{T} \int_{0}^{T} p_{i}^{p s}(t) d t, \\
& \overline{\mathcal{J}_{i j}^{p s}}=\frac{1}{T} \int_{0}^{T} \mathcal{J}_{i j}^{p s}(t) d t, \\
& \overline{\sigma_{i j}^{p s}}=\frac{1}{T} \int_{0}^{T} \sigma_{i j}^{p s}(t) d t .
\end{aligned}
$$

The problem that we wish to study can now be formulated as follows.

Problem formulation.-Given a time-independent rate matrix $\mathcal{R}$ corresponding to steady-state quantities $\vec{p}^{s s}, \mathcal{J}^{s s}$, and $\sigma^{s s}$, construct a time-periodic detailed-balance rate matrix $\mathcal{W}(t)$ whose periodic state is described by the same quantities, after averaging over time:

$$
\overline{p_{i}^{p s}}=p_{i}^{s s}, \quad \overline{\mathcal{J}_{i j}^{p s}}=\mathcal{J}_{i j}^{s s}, \quad \overline{\sigma_{i j}^{p s}}=\sigma_{i j}^{s s} .
$$

We denote the above problem as the "forward" problem. We are also interested in the "inverse" problem: Given a time-dependent detailed balance rate matrix $\mathcal{W}(t)$ corresponding to the time-averaged quantities $\overline{p_{i}^{p s}}, \overline{\mathcal{J}_{i j}^{p s}}$, and $\overline{\sigma_{i j}^{p s}}$, we want to construct a time-independent rate matrix $\mathcal{R}$ such that Eq. (13) holds. As we discuss in more detail below, the solution of the inverse problem follows directly from known results; therefore, we focus mainly on the forward problem in this paper.

When $\mathcal{R}$ and $\mathcal{W}(t)$ give rise to dynamics that satisfy Eq. (13), we say that the stochastic pump "mimics" the nonequilibrium steady state, and vice versa.

\section{B. Two useful decompositions}

The two well-known decompositions described belowthe first, an algebraic decomposition of rate matrices, and the second, a topological decomposition of the connectivity graph-will be extensively used in what follows.

\section{Rate matrix decomposition}

The following (unique) decomposition of any rate matrix $\mathcal{R}$, obtained by Zia and Schmittmann [38], will prove to be useful:

$$
\mathcal{R}=\left(\mathcal{S}+\frac{1}{2} \mathcal{J}^{s s}\right) \mathcal{P}^{-1} .
$$

Here, the multiplication is an ordinary matrix multiplication, and $\mathcal{S}$ is a symmetric matrix whose elements in each column add up to zero, with negative entries only on the diagonal. $\mathcal{J}^{s s}$ is the antisymmetric current matrix defined in Eq. (2), and $\mathcal{P}=\operatorname{diag}\left(\vec{p}^{s s}\right)$ is a diagonal matrix with elements $\mathcal{P}_{i i}=p_{i}^{s s}$.

An immediate corollary of Eq. (14) is the following statement: If a rate matrix $\mathcal{R}$ is the product of a symmetric rate matrix $\mathcal{S}$ and the inverse of a diagonal matrix $\mathcal{P}$ (with positive diagonal entries summing to unity), then $\mathcal{R}$ satisfies detailed balance; i.e., there are no currents in the stationary state.

\section{Cycle decomposition}

The currents that characterize a NESS are, in general, not independent of one another, as they must satisfy constraints arising from the conservation of probability. These constraints embody Kirchoff's law of currents. The cycle decomposition method provides a convenient tool to account for these constraints [36]. Briefly, in a connected network with $N$ nodes and $E$ edges, the conservation of probability imposes $N-1$ constraints among the $E$ currents (one current per edge). It is then convenient to identify $C=E-N+1$ fundamental currents, using the following 


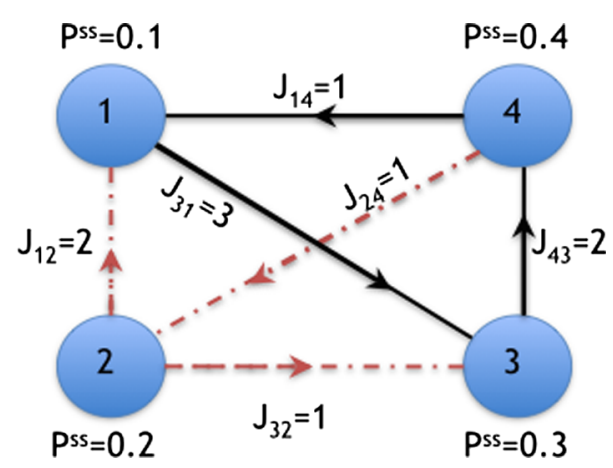

FIG. 2. A concrete example of a NESS for which we build an equivalent stochastic pump. In this example, $\mathcal{R}_{i j} \neq 0$ for all $i, j$. The spanning tree was chosen to be the 2-1, 2-3, and 2-4 edges (dashed red lines), and the fundamental currents are the currents along the 1-4, 1-3, and 3-4 edges (solid black lines). In this system, there are two current loops: $1 \rightarrow 3 \rightarrow 4 \rightarrow 1$ and $2 \rightarrow 3 \rightarrow 4 \rightarrow 2$.

procedure. First, we build a connectivity graph for the system, as described above and illustrated in Fig. 2 for the case of four nodes and six edges (hence, $C=3$ ). We then construct a maximal spanning tree, by removing $C$ edges without breaking the connectivity of the graph; this tree, illustrated by the red dash-dotted lines in Fig. 2, has no cycles. In the context of the original graph, the $C$ edges that are removed to form the spanning tree are called fundamental edges. The currents along these edges are the fundamental currents (the black arrows in the figure), and the currents along the edges of the tree are the spanning-tree currents (the red arrows in the figure).

For NESS, the steady-state currents along the $C$ fundamental edges can take on any values, independently of one another. However, once these fundamental currents are set, the spanning-tree currents are then uniquely determined by conservation of probability: The sum of incoming and outgoing currents at each node must vanish in the steady state. Therefore, the number of degrees of freedom in the matrix $\mathcal{J}^{s s}$ is not $\left(n^{2}-n\right) / 2$ as for an arbitrary antisymmetric matrix but is determined by the graph topology.

Unlike NESS, for stochastic pumps the fundamental currents at each moment do not fix the currents on the spanning-tree edges since probabilities can temporarily accumulate on the vertices (Kirchoff's current law does not apply at every instant of time). However, $\overline{\mathcal{J}^{p s}}$ on the fundamental edges does dictate $\overline{\mathcal{J}^{p s}}$ on the spanning tree since the average probabilities are conserved.

\section{MAPPING SP TO NESS}

In this section, we consider the inverse problem defined at the end of Sec. II A, which conceptually is the simpler direction: Given a time-dependent periodic rate matrix $\mathcal{W}(t)$, how do we construct a time-independent rate matrix $\mathcal{R}$ whose steady-state properties satisfy Eq. (13)?
To construct $\mathcal{R}$, we first solve for the periodic averages $\overline{p_{i}^{p s}}, \overline{\mathcal{J}_{i j}^{p s}}$, and $\overline{\sigma_{i j}^{p s}}$ associated with $\mathcal{W}(t)$. These can be calculated by obtaining the periodic solution of the master equation, $p_{i}^{p s}(t)$, and then plugging this solution into Eq. (12). In most cases, finding the periodic solution must be done numerically.

Once $\overline{p_{i}^{p s}}, \overline{\mathcal{J}_{i j}^{p s}}$, and $\overline{\sigma_{i j}^{p s}}$ are known, we must build a rate matrix $\mathcal{R}$ whose steady-state properties, $\vec{p}^{s s}, \mathcal{J}^{s s}$, and $\sigma^{s s}$, satisfy Eq. (13). A nice consequence of Eq. (14) is that if all the currents along the graph edges are nonzero, then the quantities $\vec{p}^{s s}, \mathcal{J}^{s s}$, and $\sigma^{s s}$ uniquely determine $\mathcal{R}$. We express this relationship by the shorthand notation

$$
\left\{\vec{p}^{s s}, \mathcal{J}^{s s}, \sigma^{s s}\right\} \Rightarrow \mathcal{R} \text {. }
$$

To see this, we use Eq. (14) to write the entropy production rates as

$$
\sigma_{i j}^{s s}=\mathcal{J}_{i j}^{s s} \log \frac{\mathcal{S}_{i j}+\frac{1}{2} \mathcal{J}_{i j}^{s s}}{\mathcal{S}_{i j}-\frac{1}{2} \mathcal{J}_{i j}^{s s}} .
$$

By this equation, the elements of $\mathcal{J}^{s s}$ and $\sigma^{s s}$ uniquely determine $\mathcal{S}$, if the currents are nonzero:

$$
\left\{\mathcal{J}^{s s}, \sigma^{s s}\right\} \Rightarrow \mathcal{S}
$$

If some of the currents are zero, then the corresponding elements of $\mathcal{S}$ are not uniquely determined from the currents and entropy production alone, and they can be arbitrarily chosen. Once $\mathcal{S}$ has been obtained consistently with $\mathcal{J}^{s s}$ and $\sigma^{s s}$, it can be combined with $\mathcal{P}$ and $\mathcal{J}^{s s}$, via Eq. (14), to give $\mathcal{R}$.

In the remainder of this paper, we address the forward problem, namely, how to construct, for a given NESS, a mimicking SP protocol. While this problem is conceptually more complicated than the inverse problem discussed above, it turns out that it is computationally simpler and does not require any solution of differential equations.

\section{KEY IDEAS}

Here, we establish three technical results that will play a crucial role in the construction of $\mathcal{W}(t)$.

\section{A. No current loops in detailed balance systems}

A system satisfying detailed balance has nonvanishing currents when the instantaneous probability distribution differs from the equilibrium state of the instantaneous rate matrix. These currents, however, cannot form a current loop. In other words, no loop $i, j, k, \ldots, m, i$ on the graph associated with the system can have all the currents oriented in the same direction around the loop. As an example of a current loop, consider the system described in Fig. 2. No detailed balance system can have instantaneous currents equal to the currents in the loop $1 \rightarrow 3 \rightarrow 4 \rightarrow 1$ 
(or in the loop $2 \rightarrow 3 \rightarrow 4 \rightarrow 2$ ) since they all have the same orientation.

To see why a current loop is inconsistent with detailed balance, let us consider the currents along the edges of a loop $i, j, k, \ldots, m, i$. Using Eq. (14) to decompose the detailed balance rate matrix $\mathcal{W}=\mathcal{S} \Pi^{-1}$, the currents generated by a distribution $\vec{q}$ satisfy

$$
\begin{gathered}
\frac{\mathcal{J}_{i j}}{\mathcal{S}_{i j}}=\pi_{j}^{-1} q_{j}-\pi_{i}^{-1} q_{i}, \\
\frac{\mathcal{J}_{j k}}{\mathcal{S}_{j k}}=\pi_{k}^{-1} q_{k}-\pi_{j}^{-1} q_{j}, \\
\vdots \\
\frac{\mathcal{J}_{m i}}{\mathcal{S}_{m i}}=\pi_{i}^{-1} q_{i}-\pi_{m}^{-1} q_{m} .
\end{gathered}
$$

Summing these equations, we get that $\sum(\mathcal{J} / \mathcal{S})=0$ around the loop. This means that not all the currents can have the same sign since each $\mathcal{S}_{i j}>0$. Therefore, there are no current loops. (A similar argument was used in Ref. [20]).

By contrast, the steady-state currents of a NESS must form at least one current loop. To see this, just choose a site (denoted by $i$ ) through which some of the steady-state currents flow. Conservation of probability implies that at least one of these currents is going out of site $i$, so we choose such a current, say, from $i$ into site $j$. Now, from site $j$ again, there is at least one current going out, say, to site $k$. Following the same argument, from any site, we can "flow" with a current into a new site, but since the number of sites is finite, after no more than $n$ such steps, we must come back to a site we already visited. Therefore, there must exist at least one current loop.

\section{B. Transformation for the diagonal part of the decomposition of $\mathcal{W}$}

Suppose we have a detailed balance rate matrix $\hat{\mathcal{W}}$ and two instantaneous probability distributions $\vec{q}$ and $\vec{p}$, neither of which necessarily corresponds to a stationary distribution. We would like to transform $\hat{\mathcal{W}}$ into a different detailed balance rate matrix, $\mathcal{W}$, such that $\hat{\mathcal{W}} \vec{q}=\mathcal{W} \vec{p}$. This is achieved by the following transformation:

$$
\mathcal{W}=\hat{\mathcal{W}} \mathcal{Q} \mathcal{P}^{-1},
$$

where $\mathcal{P}$ and $\mathcal{Q}$ are the diagonal matrices corresponding to $\vec{p}$ and $\vec{q}$, respectively. Using indices, this reads

$$
\mathcal{W}_{i j}=\hat{\mathcal{W}}_{i j} q_{j} p_{j}^{-1} .
$$

The transformation given by Eq. (21a) affects only the diagonal part of the decomposition and has the following properties:
(1) If $\hat{\mathcal{W}}$ satisfies the detailed balance condition, then so does $\mathcal{W}$. This can be seen by using the decomposition of $\hat{\mathcal{W}}$ as in Eq. (14) in the above transformation and noting that $\mathcal{W}$ is a symmetric rate matrix times a diagonal matrix; therefore, it has no currents in its steady state.

(2) The instantaneous currents of a system described by $\hat{\mathcal{W}}$ with probabilities $\vec{q}$ are the same as those of a system described by $\mathcal{W}$ with the probabilities $\vec{p}$. This follows by substituting Eq. (21b) into Eq. (7).

(3) From Eqs. (8) and (21b), it follows that the instantaneous entropy production rates along each edge $\left(\sigma_{i j}\right)$ for a system described by $\hat{\mathcal{W}}$ with probabilities $\vec{q}$ and for a system described by $\mathcal{W}$ with probabilities $\vec{p}$ are the same.

The significance of this transformation can be stated as follows. If we have a rate matrix $\hat{\mathcal{W}}$ and probabilities $\vec{q}$, which produce instantaneous currents $\mathcal{J}$ and entropy production rates $\sigma$, then for any other probability distribution $\vec{p}$, we can construct the rate matrix $\mathcal{W}$ that generates the same $\mathcal{J}$ and $\sigma$.

\section{Transformation for the symmetric part of the decomposition of $\mathcal{W}$}

Currents arise in a system described by a detailed balance $\mathcal{W}$ when the instantaneous probability distribution $\vec{p}$ differs from the equilibrium distribution $\vec{\pi}$. We next show how to vary the magnitudes of these currents (but not their directions) while keeping $\vec{p}$ and $\vec{\pi}$ fixed. As the directions of the currents do not vary under this transformation, no loops can be formed in accordance with the "No loop condition" in Sec. IVA.

Let us use the decomposition $\mathcal{W}=\mathcal{S} \Pi^{-1}$, where $\mathcal{S}$ is symmetric and $\Pi=\operatorname{diag}(\vec{\pi})$. The currents are then given by

$$
\mathcal{J}_{i j}=\mathcal{S}_{i j}\left(\pi_{i}^{-1} p_{j}-\pi_{j}^{-1} p_{i}\right) .
$$

We see that by varying $\mathcal{S}_{i j}$, we vary the magnitude of the current $\mathcal{J}_{i j}$, but not its sign, since $\mathcal{S}_{i j} \geq 0$.

This transformation is complementary to the one given by Eq. (21): It enables us to adjust the currents and entropy production rates while keeping the probabilities $\vec{p}$ and $\vec{\pi}$ fixed, by tuning the symmetric part of $\mathcal{W}$ in the decomposition given by Eq. (14). By contrast, with the previous transformation, we can vary the probabilities $\vec{p}$ and $\vec{\pi}$ at fixed currents and entropy production rates, by tuning the diagonal part of $\mathcal{W}$.

\section{CONSTRUCTION OF THE PUMPING PROTOCOL}

Given a rate matrix $\mathcal{R}$, one can calculate its steady state $\vec{p}^{s s}$ (the null eigenvector of $\mathcal{R}$ ) and thus $\mathcal{J}^{s s}$ and $\sigma^{s s}$ using Eqs. (2) and (3). Our goal is to construct a periodic 
pumping protocol - a time-dependent detailed balance rate matrix $\mathcal{W}(t)$ with some period $T$-such that Eq. (13) holds, in other words, a SP that mimics the NESS. For simplicity, we assume that there are no edges along which the steadystate currents are zero. The case involving zero currents along some edges is analyzed in the Appendix B.

We note that, in general, there are many SPs that mimic any specific NESS - the mapping is not one to one. Out of the many SPs that mimic the NESS, we choose one using a relatively simple construction, yet generic enough to mimic any NESS. Naively, we would like to have a SP whose periodic state gives rise to time-independent quantities, $p_{i}^{p s}(t)=p_{i}^{s s}, \mathcal{J}_{i j}^{p s}(t)=\mathcal{J}_{i j}^{s s}$, and $\sigma_{i j}^{p s}(t)=\sigma_{i j}^{s s}$. Such a construction is, unfortunately, impossible. This can be seen from the result of Sec. IVA, which states that for all $t$, $\mathcal{J}_{i j}^{p s}(t)$ cannot have any current loops, whereas $\mathcal{J}_{i j}^{s s}$ must have at least one current loop. Thus, we can only hope to achieve a mapping between the time-averaged quantities associated with the SP and those of the NESS, as in Eq. (13). This also implies that at least some of the currents of the SP must be time dependent.

Though it was the simplest we could find, the construction described below is nevertheless somewhat convoluted. We therefore first give an overview before proceeding to the detailed description. The main reason for the complication is the fact that the periodic solution $p_{i}^{p s}(t)$ is a highly nontrivial function of the pumping protocol $\mathcal{W}(t)$. To avoid this complication, we simultaneously construct both the pumping protocol $\mathcal{W}(t)$ and its periodic solution $p_{i}^{p s}(t)$. This is achieved in five steps.

In the first step, described in Sec. VA, we divide the pumping protocol temporal interval of duration $T$ into two equal half-intervals, designated as $a$ and $b$. We then assign an arbitrary fixed detailed-balance matrix $\tilde{\mathcal{W}}^{a}$ and an arbitrary fixed probability distribution $\vec{q}^{a}$, to be associated with the first half-period of driving. We refer to $\tilde{\mathcal{W}}^{a}$ and $\vec{q}^{a}$ together as the seed for that half-cycle, and these quantities will be used to set the current directions during that time interval. Next, the seed and current directions for the first half-cycle are used to assign a seed $\left(\tilde{\mathcal{W}}^{b}, \vec{q}^{b}\right)$ and current directions for the second half-cycle. The current directions during the first half-cycle are opposite of those of the second half-cycle, and both sets are, by construction, consistent with the no-current-loop condition in Sec. IVA.

In the next two steps, we use the seeds to construct fixed sets of currents $\left(\mathcal{J}_{i j}^{a}\right.$ and $\left.\mathcal{J}_{i j}^{b}\right)$ and entropy production rates $\left(\sigma_{i j}^{a}\right.$ and $\left.\sigma_{i j}^{b}\right)$ for the first and second halves of the cycle, whose averages over the two halves are equal to the steadystate values that we wish to mimic:

$$
\mathcal{J}_{i j}^{s S}=\frac{1}{2}\left(\mathcal{J}_{i j}^{a}+\mathcal{J}_{i j}^{b}\right), \quad \sigma_{i j}^{S S}=\frac{1}{2}\left(\sigma_{i j}^{a}+\sigma_{i j}^{b}\right)
$$

for all $i \neq j$. This is done first for the fundamental currents in Sec. VB and then for the spanning-tree currents in Sec. V C.

Up to this point, the currents and entropy production rates for the two half-cycles have been constructed from the initial, arbitrary seeds, but the corresponding time-periodic rate matrices $\mathcal{W}^{a}(t)$ and $\mathcal{W}^{b}(t)$ that actually generate these currents and entropies are not yet known. In the fourth step, described in Sec. V D, we use the transformation of Sec. IV $\mathrm{C}$ to adjust the symmetric part of the seed matrices, $\tilde{\mathcal{W}}^{a, b}$, arriving at new, time-independent rate matrices $\hat{\mathcal{W}}^{a, b}$ that produce the desired currents $\mathcal{J}^{a, b}$, for the seed probabilities $\vec{q}^{a, b}$. These currents, together with the desired averaged probabilities $\overline{p_{i}^{p s}}$, fix $p_{i}^{p s}(t)$. In the last step (Sec. V E), we use the transformation of Sec. IV B, together with the symmetric parts of $\hat{\mathcal{W}}^{a, b}$, to construct time-dependent rate matrices $\mathcal{W}^{a}(t)$ and $\mathcal{W}^{b}(t)$ for which $p_{i}^{p s}(t)$ is the periodic solution of the master equation.

In the specific protocol described below, the entries of the matrix $\mathcal{W}(t)$ are not continuous functions of time, as they have discontinuities between the two $T / 2$ intervals. These discontinuities are not essential and can be removed at the expense of making the construction less transparent.

For clarity of presentation, some of the formal definitions of the construction are followed by a concrete application to the example of the four-state system described in Fig. 2. In this example, the NESS system has four states with $\vec{p}^{s s}=(0.1,0.2,0.3,0.4)$. The fundamental currents were chosen to be $\mathcal{J}_{31}^{s s}=3, \mathcal{J}_{43}^{s s}=2$, and $\mathcal{J}_{14}^{s s}=1$. The currents for the spanning-tree edges are then dictated by Kirchoff's law-the sum of currents in each vertex must be zero. The corresponding current matrix is

$$
\mathcal{J}^{s s}=\left(\begin{array}{cccc}
0 & 2 & -3 & 1 \\
-2 & 0 & 1 & 1 \\
3 & -1 & 0 & -2 \\
-1 & -1 & 2 & 0
\end{array}\right) .
$$

Finally, we choose the entropy production rate to be 1 along all the edges: $\sigma_{i j}=1$ for all $i \neq j$. Using Eq. (16), the matrix $\mathcal{S}$ can be calculated, and it is given in Eq. (A2) in Appendix A. The matrix $\mathcal{R}$ giving rise to this particular NESS can be constructed using Eq. (14).

\section{A. Step 1: Choosing the seed}

In what follows, superscripts $a$ and $b$ indicate quantities associated with the first and second halves of the period, respectively.

Our first step is to choose an arbitrary detailed balance matrix on the graph. This is done by choosing an equilibrium state for the first half-period $\vec{\pi}^{a}$ and a symmetric rate matrix $\tilde{\mathcal{S}}$ from which we compose $\tilde{\mathcal{W}}^{a}=\tilde{\mathcal{S}}\left(\Pi^{a}\right)^{-1}$, where 
$\Pi^{a}=\operatorname{diag}\left(\vec{\pi}^{a}\right)$. Next, we choose a fixed probability distribution $\vec{q}^{a} \neq \vec{\pi}^{a}$ that satisfies

$$
\left|\log \frac{\pi_{i}^{a} q_{j}^{a}}{q_{i}^{a} \pi_{j}^{a}}\right|<\left|\log \frac{\mathcal{R}_{i j} p_{j}^{s s}}{\mathcal{R}_{i j} p_{i}^{s s}}\right|
$$

for any $i \neq j$. It is always possible to satisfy this condition by choosing $\vec{q}^{a}$ close enough to $\vec{\pi}^{a}$. As we see in the next section, this condition is necessary for the consistency of our construction.

For the second half of the period, we replace $\vec{\pi}^{a}$ and $\vec{q}^{a}$ by vectors with components $\pi_{i}^{b}=1 / \pi_{i}^{a}$-from which we construct $\tilde{\mathcal{W}}^{b}=\tilde{\mathcal{S}}\left(\Pi^{b}\right)^{-1}$-and $q_{i}^{b}=1 / q_{i}^{a}$. The two probability vectors $\vec{\pi}^{b}$ and $\vec{q}^{b}$ are not normalized, but as will become clear, this normalization does not play any role; it will prove to be simpler to work with these unnormalized vectors. We note that the currents generated by $\tilde{\mathcal{W}}^{a}$ and $\vec{q}^{a}$,

$$
\tilde{\mathcal{J}}_{i j}^{a}=\tilde{\mathcal{S}}_{i j}\left(\pi_{j}^{a} q_{j}^{a}-\pi_{i}^{a} q_{i}^{a}\right),
$$

have, on each edge, opposite signs to those generated by $\tilde{\mathcal{W}}^{b}$ and $\vec{q}^{b}$, given by

$$
\tilde{\mathcal{J}}_{i j}^{b}=\tilde{\mathcal{S}}_{i j}\left(\frac{1}{\pi_{j}^{a} q_{j}^{a}}-\frac{1}{\pi_{i}^{a} q_{i}^{a}}\right) .
$$

The values of $\tilde{\mathcal{J}}_{i j}^{a, b}$ will not explicitly be used in what follows-only their directions, which by construction are consistent with detail balance. We additionally note that

$$
\log \frac{\pi_{i}^{a} q_{j}^{a}}{q_{i}^{a} \pi_{j}^{a}}=-\log \frac{\pi_{i}^{b} q_{j}^{b}}{q_{i}^{b} \pi_{j}^{b}}
$$

Normalizing the probabilities $\vec{\pi}^{b}$ and $\vec{q}^{b}$ does not change Eq. (28), nor the sign of $\tilde{\mathcal{J}}_{i j}^{b}$ in Eq. (27). Thus, working with the non-normalized probabilities is justified.

To illustrate this part of the construction with our fourstate example, we choose

$$
\tilde{\mathcal{S}}=\left(\begin{array}{cccc}
-3 & 1 & 1 & 1 \\
1 & -3 & 1 & 1 \\
1 & 1 & -3 & 1 \\
1 & 1 & 1 & -3
\end{array}\right), \quad \vec{\pi}^{a}=\left(\begin{array}{c}
1 / 4 \\
1 / 4 \\
1 / 4 \\
1 / 4
\end{array}\right) .
$$

We emphasize that both $\tilde{\mathcal{S}}$ and $\vec{\pi}^{a}$ are arbitrary.

We next note that $\min _{i j}\left[\log \mathcal{R}_{i j} p_{j}^{s s}-\log \mathcal{R}_{j i} p_{i}^{s s}\right]=1 / 3$. Therefore, if we choose $\vec{q}^{a}$ such that $\left|\log \pi_{i}^{a}-\log q_{i}^{a}\right|<$ $1 / 6$, then Eq. (25) is satisfied. Any vector close enough to $\vec{\pi}^{a}$ will do. As an example, we use $\vec{q}^{a}=(0.23,0.24,0.26,0.27)$. In the second half of the period, these correspond to $\vec{\pi}^{b}=(4,4,4,4)$ and $\vec{q}^{b}=$ $(4.3478,4.1667,3.8462,3.7037)$.

\section{B. Step 2: Fundamental edge currents}

In this step, we set the currents along the fundamental edges to be constant during each of the two half cycles, such that their time averages (the average between the first and second halves) is equal to $\mathcal{J}^{s s}$, and the time average of the entropy production rates is equal to $\sigma^{s s}$. Moreover, we choose these fundamental currents to have the same directions as the fundamental currents in $\tilde{\mathcal{J}}^{a}$ during the first half-cycle and to be reversed in direction during the second half-cycle.

We first make sure that for each fundamental edge, the average of $\mathcal{J}_{i j}^{a}$ and $\mathcal{J}_{i j}^{b}$, and therefore the time-averaged current, is exactly $\mathcal{J}_{i j}^{s s}$. To that effect, we introduce the following rules:

(i) If the direction of the NESS current, $\mathcal{J}_{i j}^{S S}$, is the same as the direction of $\tilde{\mathcal{J}}_{i j}^{a}$ defined above, then we set in the first half of the period $\mathcal{J}_{i j}^{a}=\left(2+\alpha_{i j}\right) \mathcal{J}_{i j}^{s s}$ and in the second half of the period $\mathcal{J}_{i j}^{b}=-\alpha_{i j} \mathcal{J}_{i j}^{s s}$, where $\alpha_{i j}$ are positive and will be determined below.

(ii) If the direction of the NESS current, $\mathcal{J}_{i j}^{s s}$, is not the same as the direction of $\tilde{\mathcal{J}}_{i j}^{a}$, then we set in the first half of the period $\mathcal{J}_{i j}^{a}=-\alpha_{i j} \mathcal{J}_{i j}^{s S}$ and in the second half $\mathcal{J}_{i j}^{b}=\left(2+\alpha_{i j}\right) \mathcal{J}_{i j}^{s s}$.

According to this rule, the directions of the currents during the first half of the period are the same as those of $\tilde{\mathcal{J}}_{i j}^{a}$ and are opposite to those in the second half. Moreover, by the above construction, the time-averaged currents on the fundamental edges have the required values

$$
\frac{1}{2}\left(\mathcal{J}_{i j}^{a}+\mathcal{J}_{i j}^{b}\right)=\mathcal{J}_{i j}^{s s}
$$

Next, we determine the values of the $\alpha_{i j}$ 's so as to satisfy the requirement on the entropy production rates. Assuming, for the moment, that the probability distributions in the first and second halves of the period are given by $\vec{q}^{a}$ and $\vec{q}^{b}$ and that the equilibrium distributions of the detailed balance matrices during the first and second halves of the period are given by $\vec{\pi}^{a}$ and $\vec{\pi}^{b}$, respectively, then the entropy production rates with the currents $\mathcal{J}_{i j}^{a}$ and $\mathcal{J}_{i j}^{b}$ during the two halves of the cycle are given by

$$
\begin{aligned}
\sigma_{i j}^{a} & =\mathcal{J}_{i j}^{a} \log \frac{\pi_{j}^{a} q_{j}^{a}}{\pi_{i}^{a} q_{i}^{a}}, \\
\sigma_{i j}^{b} & =\mathcal{J}_{i j}^{b} \log \frac{\pi_{j}^{b} q_{j}^{b}}{\pi_{i}^{b} q_{i}^{a}} .
\end{aligned}
$$

Substituting in these equations $\mathcal{J}_{i j}^{a}$ and $\mathcal{J}_{i j}^{b}$ in terms of $\alpha_{i j}$ and demanding that $\frac{1}{2}\left(\sigma_{i j}^{a}+\sigma_{i j}^{a}\right)=\sigma_{i j}^{s S}$, we get an equation for $\alpha_{i j}$ whose solution is 


$$
\alpha_{i j}=\left|\left(\log \frac{\pi_{i}^{a} q_{j}^{a}}{q_{i}^{a} \pi_{j}^{a}}\right)^{-1} \log \frac{\mathcal{R}_{i j} p_{j}^{s s}}{\mathcal{R}_{i j} p_{i}^{s s}}\right|-1
$$

Equation (25) ensures that indeed $\alpha_{i j}>0$.

To illustrate this step, we examine the signs of the currents generated by the matrix $\tilde{\mathcal{W}}^{a}=\tilde{\mathcal{S}}\left(\Pi^{a}\right)^{-1}$ and the probability $\vec{q}^{a}$, denoted by $\tilde{\mathcal{J}}^{a}$, on the fundamental edges:

$$
\begin{aligned}
& \operatorname{sign}\left(\tilde{\mathcal{J}}_{13}^{a}\right)=+1, \\
& \operatorname{sign}\left(\tilde{\mathcal{J}}_{14}^{a}\right)=+1, \\
& \operatorname{sign}\left(\tilde{\mathcal{J}}_{34}^{a}\right)=+1 .
\end{aligned}
$$

While the direction of the current along the 1-4 edge is the same as the current orientation of $\mathcal{J}^{s s}$, for the other two fundamental edges, the directions of $\mathcal{J}^{a}$ and $\mathcal{J}^{s s}$ are not the same.

We next solve Eq. (32) for $\alpha_{i j}$. The explicit expression, as well as the currents, is given in the Appendix A. Figure 3 shows the currents on the first half-period (left) and second half-period (right). Note that (i) the direction of the currents along each edge is opposite in the two halves of the period, and (ii) as discussed above, in the first half-period the direction of the current along the 1-4 edge is the same as that of Fig. 2, but the direction of currents along the other fundamental edges is different from that of Fig. 2, and (iii) there are no current loops in Fig. 3.

\section{Step 3: Spanning-tree currents}

So far, we have described how to construct the fundamental edge currents. Next, we discuss the currents along the edges of the spanning tree. For both the first and the second half-periods, we impose the following two constraints:

(1) The sum of currents feeding into any site $i$ during the first half-period must be equal to minus the same quantity during the next half-period:

$$
\sum_{j} \mathcal{J}_{i j}^{a}=-\sum_{j} \mathcal{J}_{i j}^{b}
$$

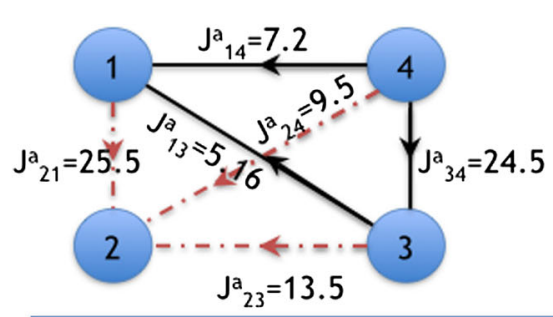

First half cycle
These constraints ensure that we indeed have a periodic time evolution: $\Delta p_{i}=\int_{0}^{T} \partial_{t} p_{i} d t=$ $(T / 2) \sum_{j}\left(\mathcal{J}_{i j}^{a}+\mathcal{J}_{i j}^{b}\right)=0$. Note that these are only $n-1$-independent equations since conservation of probability adds the constraint $\sum_{i j} \mathcal{J}_{i j}^{a, b}=0$.

(2) For each spanning-tree edge, we demand that

$$
\mathcal{J}_{i j}^{a} \log \frac{\pi_{i}^{a} q_{j}^{a}}{q_{i}^{a} \pi_{j}^{a}}+\mathcal{J}_{i j}^{b} \log \frac{\pi_{i}^{b} q_{j}^{b}}{q_{i}^{b} \pi_{j}^{b}}=2 \sigma_{i j}^{s s} .
$$

The number of edges in the spanning tree is $n-1$, and therefore, these are $n-1$ additional equations. They ensure that the time-averaged entropy production rate along the $i, j$ edge is the same as $\sigma_{i j}^{s s}$.

Equations (34) and (35) are 2(n-1) linear equations for $2(n-1)$ unknowns: $\mathcal{J}^{a, b}$ on the spanning tree, which has $n-1$ edges [39]. Moreover, the directions $\mathcal{J}_{i j}^{a, b}$ that solve these equations are the same as those of $\tilde{\mathcal{J}}_{i j}^{a, b}$, respectively. To see this, let us use the definition of $\vec{q}^{b}$ and $\vec{\pi}^{b}$ in the second condition above, together with the definition of $\sigma_{i j}^{s s}$ [Eq. (3)]:

$$
\left(\mathcal{J}_{i j}^{a}-\mathcal{J}_{i j}^{b}\right)=2 \mathcal{J}_{i j}^{s s}\left(\log \frac{\pi_{i}^{a} q_{j}^{a}}{q_{i}^{a} \pi_{j}^{a}}\right)^{-1} \log \frac{\mathcal{R}_{i j} p_{j}^{s s}}{\mathcal{R}_{j i} p_{i}^{s s}} .
$$

Taking the absolute value of both sides in the above equation and using Eq. (25) implies that

$$
\left|\mathcal{J}_{i j}^{a}-\mathcal{J}_{i j}^{b}\right|>2\left|\mathcal{J}_{i j}^{s s}\right| .
$$

However, by the construction of $\mathcal{J}_{i j}^{a}$ and $\mathcal{J}_{i j}^{b}$, their average is equal to the steady-state current, $\mathcal{J}_{i j}^{s s}$; therefore,

$$
\left(\mathcal{J}_{i j}^{a}+\mathcal{J}_{i j}^{b}\right) / 2=\mathcal{J}_{i j}^{s s} .
$$

Equations (37) and (38) are consistent with each other only if the sign of $\mathcal{J}_{i j}^{a}$ is opposite to that of $\mathcal{J}_{i j}^{b}$. Moreover, as $\sigma_{i j}^{s S}>0$ [this follows from Eq. (16)], the signs of $\mathcal{J}_{i j}^{a}$ and $\mathcal{J}_{i j}^{b}$ must be the same as those of $\log \pi_{i}^{a} q_{j}^{a} / q_{i}^{a} \pi_{j}^{a}$ and $\log \pi_{i}^{b} q_{j}^{b} / q_{i}^{b} \pi_{j}^{b}$, respectively; otherwise, the left-hand side
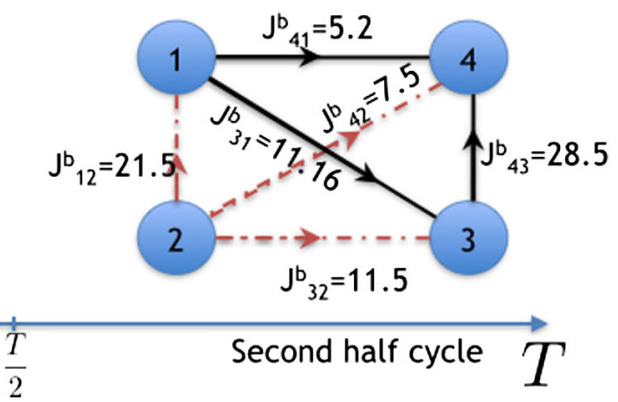

FIG. 3. The currents of the stochastic pump constructed to mimic the NESS in Fig.(2). The pumped currents during the first (left) and second (right) half-periods are shown. On each of the edges, the current directions in the two half periods are opposite. Note that there are no current loops in both of the half periods. 
of Eq. (35) will be negative. Therefore, it is also the same as the sign of $\tilde{\mathcal{J}}_{i j}^{a}$.

\section{Step 4: Transforming the symmetric part for the currents}

At this stage, we have constructed the time-dependent currents for both half-cycles with the same directions as the currents of $\tilde{\mathcal{W}}^{a, b}$ with $\vec{q}^{a, b}$. We can now use the transformation of the symmetric part of the decomposition of the rate matrix (defined in Sec. IV C) to adjust the symmetric part of $\tilde{\mathcal{W}}$ such that the currents generated by the transformed matrix and the seed probabilities $\vec{q}^{a, b}$ have the constructed values

$$
\begin{aligned}
\mathcal{S}_{i j}^{a} & =\frac{\mathcal{J}_{i j}^{a}}{\left(\pi_{j}^{a}\right)^{-1} q_{j}^{a}-\left(\pi_{i}^{a}\right)^{-1} q_{i}^{a}}, \\
\mathcal{S}_{i j}^{b} & =\frac{\mathcal{J}_{i j}^{b}}{\left(\pi_{j}^{b}\right)^{-1} q_{j}^{b}-\left(\pi_{i}^{b}\right)^{-1} q_{i}^{b}} .
\end{aligned}
$$

Importantly, all these off-diagonal elements are positive. This follows from the fact that the denominators are just $\tilde{\mathcal{J}}_{i j}^{a, b} / \tilde{\mathcal{S}}_{i j}$, but by our construction, the sign of $\mathcal{J}_{i j}^{a, b}$ is the same as that of $\tilde{\mathcal{J}}_{i j}^{a, b}$. The diagonal elements of $\mathcal{S}^{a, b}$ are now determined by the requirement that the sum of each of the columns is zero.

\section{E. Step 5: Forming a solution to the master equation}

We have now arrived at the matrices $\hat{\mathcal{W}}^{a, b}=$ $\mathcal{S}^{a, b}\left(\Pi^{a, b}\right)^{-1}$. These are the transformed seed matrices, which have been constructed (by tuning the elements of $\left.\mathcal{S}^{a, b}\right)$ so as to produce the desired currents and entropy production rates when the probability vectors are $\vec{q}^{a, b}$. However, the time average of $\vec{q}^{a, b}$ is not $\vec{p}^{s s}$, and in fact, $\vec{q}^{a, b}$ are not solutions of the master equation: $\partial_{t} \vec{q}^{a, b} \neq \hat{\mathcal{W}}^{a, b} \vec{q}^{a, b}$. To remedy this situation, we use the transformation of the diagonal part of the decomposition of $\hat{\mathcal{W}}$, defined in Sec. IV B.

First, we want the time-averaged probabilities to be equal to $\vec{p}^{s s}$. Second, we already know what $\partial_{t} p_{i}$ should be, in terms of the desired currents $\mathcal{J}^{a, b}$. Namely, $\partial_{t} p_{i}=\sum_{j} \mathcal{J}_{i j}$. Therefore, we do the following:

(1) Calculate $\vec{m}^{a, b}=\partial_{t} \vec{p}=\hat{\mathcal{W}}^{a, b} \vec{q}^{a, b}$. These are the temporal slopes of the probabilities that solve the master equation, during the first and second halves of the period.

(2) Choose $T$ such that $0<p_{i}^{s s} \pm(T / 4) m_{i}^{a, b}<1$ for any $i$. This choice ensures that the probabilities stay bounded between 0 and 1 , and is always possible by taking $T$ to be small enough.

(3) Construct $p_{i}^{a}(t)=p_{i}^{s s}-(T / 4) m_{i}^{a}+m_{i}^{a} t \quad$ and $p_{i}^{b}(t)=p_{i}^{s s}-(3 T / 4) m_{i}^{b}+m_{i}^{b} t$. These are the solutions of the master equation in the first and second halves of the period, respectively. It follows from Eq. (34) that $m_{i}^{b}=-m_{i}^{a}$. This further implies that the probabilities defined above are continuous functions of time, i.e., $p_{i}^{a}(T / 2)=p_{i}^{b}(T / 2)$ and $p_{i}^{a}(0)=p_{i}^{b}(T)$, as they should be.

(4) From the above, we define

$$
\mathcal{W}(t)= \begin{cases}\mathcal{S}^{a}\left(\Pi^{a}\right)^{-1} \mathcal{Q}^{a}\left(\mathcal{P}^{a}\right)^{-1}(t) & t<T / 2 \\ \mathcal{S}^{b}\left(\Pi^{b}\right)^{-1} \mathcal{Q}^{b}\left(\mathcal{P}^{b}\right)^{-1}(t) & t>T / 2\end{cases}
$$

The matrix $\mathcal{W}(t)$ has all the periodic state averages we demand, and its periodic state solutions in the two halves of the period are, by construction, $p^{a, b}(t)$.

For our example, we calculate the slopes $\partial_{t} \vec{p}$ on the two half-cycles by proper summation of the currents: $\partial_{t} \vec{p}^{a}=$ $(37.89,-2.51,5.85,-41.22)$ and $\partial_{t} \vec{p}^{b}=(-37.89,2.51$, $-5.85,41.22)$, which, as expected, are equal in magnitude and opposite in sign. To satisfy $0<p_{i}(t)<1$, we must choose $T$ small enough, say, $T=0.01$. Using $T$, we can obtain the time-dependent probability distributions $\vec{p}^{a, b}(t)$. These linear functions are plotted in Fig. 4. Plugging these into Eq. (40) gives $\mathcal{W}(t)$. To verify that the solution of the master equation with the constructed $\mathcal{W}(t)$ has the required properties, we solve this system numerically. The numerical results for $\vec{p}(t)$, shown as red circles in Fig. 4, agree (up to numerical error) with the analytical solution.

\section{F. Remarks}

We conclude this section with a few comments on our construction. First, the protocol presented above is clearly not unique. For example, different seeds or choices of a spanning tree result in different protocols. The nonuniqueness might be used, in principle, to match additional quantities, e.g., fluctuations around the average or the rate of decay towards the steady and periodic states. In addition,

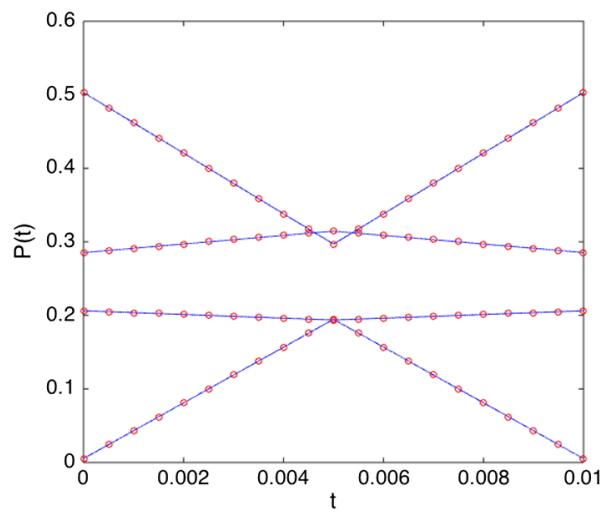

FIG. 4. $\vec{p}(t)$ of the proposed construction for our four-state example. The blue line is the exact (constructed) solution, and the red dots represent the numerical solution of the master equation. 
as mentioned previously, in our construction, $\mathcal{W}(t)$ has discontinuities between the two halves of the period. This results from discontinuities in $\mathcal{S}, \Pi$, and $\mathcal{Q}$. The discontinuities in $\mathcal{S}$ can be avoided if the currents are not taken to be fixed during the two halves of the period but changing with time, such that the currents at $t=T / 2$ vanish. The discontinuities in $\Pi$ and $\mathcal{Q}$ can be avoided if we change $\vec{q}$ as a function of time, crossing $\vec{\pi}$ at $t=T / 2$. This, however, makes the construction more cumbersome. Next, we note that in the above construction, both the symmetric and the diagonal parts of the decomposition of $\mathcal{W}$ change with time. This is known to be an essential feature of all pumping protocols [21].

Lastly, we note that for NESS, there is no minimal entropy production rate associated with a given set of currents, as is evident from Eq. (16): For any $\mathcal{J}$, the entropy production rates of the NESS can be made arbitrarily small by taking $\mathcal{S}$ to be sufficiently large [38]. Our mapping implies this is also the case for stochastic pumps: Finite currents can be pumped with arbitrarily small values of dissipation. This is surprising, though a similar result was obtained in the context of hidden, reversible pumps [28]. Stated more generally, for any connected graph, both a NESS and a SP can be constructed to have any desired set of (time-averaged) probabilities, nonzero currents, and positive entropy production rates, provided the currents obey Kirchhoff's law.

Let us now consider the construction of a stochastic pump with small entropy production rates but large currents. For the entropy production rates of the NESS to be very small with nonvanishing currents, $\log \left(\mathcal{R}_{i j} p_{j}^{s s} / \mathcal{R}_{j i} p_{i}^{s s}\right)$ must be very small, say, of order $\varepsilon$. Equation (25) then implies that $\log \left(\pi_{i}^{a} q_{j}^{a} / \pi_{j}^{a} q_{i}^{a}\right) \sim \mathcal{O}(\varepsilon)$ as well. Exponentiating this gives $\left(\pi_{i}^{a} q_{j}^{a} / \pi_{j}^{a} q_{i}^{a}\right)=1+\mathcal{O}(\varepsilon)$; thus, $\left(\pi_{j}^{a}\right)^{-1} q_{j}^{a}-\left(\pi_{i}^{a}\right)^{-1} q_{i}^{a} \sim \mathcal{O}(\varepsilon)$. By Eq. (39), this implies $\mathcal{S} \sim \mathcal{O}\left(\varepsilon^{-1}\right)$. Therefore, in stochastic pumps-as in NESS - small entropy production rates with nonvanishing currents come at the cost of large values in the symmetric part of the rate matrix. But when the elements of $\mathcal{S}$ are large, the corresponding transitions occur very rapidly. Thus, we obtain finite currents at arbitrarily low dissipation when there is a large separation of time scales, with transitions among the $n$ states of the system-and therefore relaxation to equilibrium-occurring much more rapidly than the external driving of parameters. The greater the separation of time scales, the more the stochastic pump approaches the adiabatic (quasistatic) limit [5], in which the system remains in thermal equilibrium at all times and there is no entropy production.

\section{APPLICATION: KINETIC PROOFREADING}

In the previous section, we have introduced a mapping from any NESS to a SP with the same set of averaged currents, probabilities, and entropy production rates.
However, this construction requires the ability to control all the elements of the rate matrix $\mathcal{W}(t)$, which might be experimentally challenging, or even impossible, in some applications. Can the NESS-SP mapping be achieved even with constrained $\mathcal{W}(t)$ ? The answer to this question clearly depends on the details of the constraints. Nevertheless, the tools developed so far might be useful even for constrained systems. We next demonstrate this through a concrete example: kinetic proofreading in a simple system. In this example, the constraints are set not by experimental limitations but by the structure of the problem itself. In the next subsections, we present the main idea of kinetic proofreading and how it can be achieved using NESS. We then show that despite the constraints, kinetic proofreading has a NESS-SP equivalence in terms of averaged probabilities, currents, and entropy production rates.

\section{A. Kinetic proofreading by NESS}

\section{Introduction to kinetic proofreading}

Kinetic proofreading was originally introduced by Hopfield [34] and Ninio [35] to explain the small error rates in biochemical reactions such as protein synthesis. The system in kinetic proofreading is described by a discrete-state continuous-time Markovian model. Each state in the system is associated with an energy $E_{i}$, and the transition rates between the states are of Arrhenius type,

$$
\mathcal{W}_{i j}= \begin{cases}e^{-\left(B_{i j}-E_{j}\right)} & i \neq j \\ -\sum_{k \neq j} \mathcal{W}_{i k} & i=j,\end{cases}
$$

where $B_{i j}=B_{j i}$ is the height of the barrier between the $i$ th and $j$ th states; for simplicity, we work in units where the inverse temperature is $\beta=1$. The rate matrix $\mathcal{W}$ defined above is detailed balanced, and its equilibrium distribution is given by the Boltzmann distribution,

$$
\pi_{i}=\frac{e^{-E_{i}}}{Z}, \quad \text { where } Z=\sum_{i} e^{-E_{i}} .
$$

The quantity of greatest interest in kinetic proofreading is the discrimination ratio $D$ defined as the ratio between the steady-state probability to be in one of the states, $c$ (the "correct" state), and the probability to be in some other specific state, $w$ (the "wrong" or incorrect state). The reason why $D$ is the important quantity (rather than the probability to be in the correct state) is that, in the process of protein synthesis, there is a small probability per unit time to jump from the two specific states, $w$ and $c$, into the next stage in the synthesis (which is not part of the system described so far). This probability per unit time is assumed to be small enough not to have any significant effect on the evolution of the system itself, which therefore relaxes into its steady state. As there are no direct transitions to the next stage 
from other than the $c$ or $w$ states, the error rate is equal to the ratio between the probabilities to be in states $c$ and $w$.

If the system is in equilibrium, then $D$ is given by

$$
D_{\mathrm{eq}}=\frac{\pi_{c}}{\pi_{w}}=e^{\left(E_{w}-E_{c}\right)} .
$$

This ratio depends only on the energy difference between the two states. Kinetic proofreading is a method used by nature to increase the discrimination ratio beyond its equilibrium value. The main challenge in kinetic proofreading is therefore to control the discrimination ratio without changing the energies $E_{c}$ and $E_{w}$, as well as the barrier between them; namely, these energies and barrier are given as constraints.

For simplicity, in what follows, we consider a specific example of a three-state system, shown in Fig. 5, whose states are $c, w$, and an auxiliary state denoted by $x$. This system, which is the minimal system in which detailed balance can be violated, is not the standard system for kinetic proofreading in biological context, which has five states [40]. Nevertheless, the main idea can be demonstrated through this example, which is significantly simpler.

In biological systems, kinetic proofreading is achieved through breaking detailed balance, e.g., coupling the $w \leftrightarrow x$ transition to the hydrolysis of ATP into $A D P$, whose concentrations are constantly maintained out of equilibrium. Such a coupling modifies the Arrhenius rates: Denoting the rate matrix now by $\mathcal{R}$ (as it is not detailed balanced anymore), its off-diagonal elements are the same as those of $\mathcal{W}$, i.e., $\mathcal{R}_{i j}=\mathcal{W}_{i j}$, except for the $w x$ and $x w$ rates, which become

$$
\begin{aligned}
& \mathcal{R}_{w x}=\mathcal{W}_{w x} e^{+\phi / 2}, \\
& \mathcal{R}_{x w}=\mathcal{W}_{x w} e^{-\phi / 2}
\end{aligned}
$$

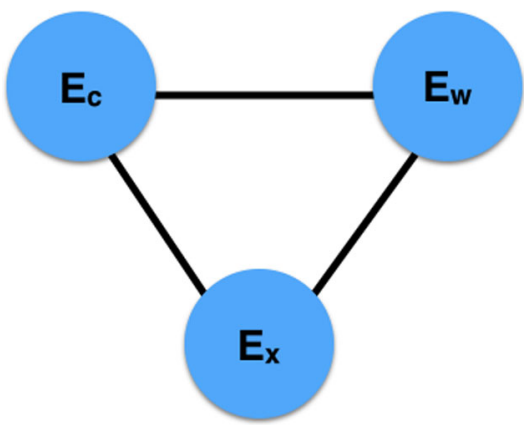

FIG. 5. A three-state system, through which we demonstrate kinetic proofreading. The system has three states: the correct state $c$, the wrong state $w$, and the auxiliary state $x$. Each state is associated with internal energies $E_{c}, E_{w}$, and $E_{x}$, respectively. Between any two states, there is a (symmetric) barrier, $B_{c w}=B_{w c}, B_{c x}=B_{x c}$, and $B_{w x}=B_{x w}$. The transition rates between the different states are given by the corresponding Arrhenius rates.
Here, the affinity $\phi$ is the Gibbs free energy released by the hydrolysis of one ATP molecule, and it can be viewed as a thermodynamic force that drives current around the loop $x \rightarrow w \rightarrow c \rightarrow x$ [36]. In principle, this affinity could have been assigned to the $x c$ edge, or split between the $x c$ and $x w$ edges, without fundamentally affecting the results. As the dynamics no longer satisfy detailed balance, the steadystate probabilities $\vec{p}^{s s}$ are not given by the Boltzmann distribution; thus, kinetic proofreading is possible.

\section{Constructing a NESS kinetic proofreading with arbitrary steady-state distribution}

Let us demonstrate that given the energies $E_{c}, E_{w}$ and the barrier $B_{c w}$, it is possible to achieve any desired probability distribution $\vec{p}^{s s}$ as the steady state of the system, by controlling the affinity $\phi$, the auxiliary state energy $E_{x}$, and the barriers between the auxiliary state and the other states $-B_{x c}$ and $B_{x w}$. In other words, we assume that the properties of the two states $c$ and $w$ and the transitions between them are given, and our goal is to show that by choosing the affinity and the auxiliary state properties, it is possible to achieve any probability distribution as the steady state of the system. To show this, we first note that there is only a single current in the system since there is only one loop. This current can be expressed [substituting $\mathcal{R}$ in Eq. (2)] in terms of the known quantities $E_{c}, E_{w}, B_{c w}$ and the desired steady-state probabilities $p_{c}^{s s}$ and $p_{w}^{s s}$ :

$$
\mathcal{J}^{s s}=e^{-B_{c w}}\left(e^{E_{c}} p_{c}^{s s}-e^{E_{w}} p_{w}^{s s}\right) .
$$

Note that the known steady-state probabilities $p_{w}^{s s}, p_{c}^{s s}$, the energies $E_{c}, E_{w}$, and the barrier $B_{c w}$ uniquely define the steady-state current $\mathcal{J}^{s s}$; namely, it cannot be tuned by changing the other parameters. In the steady state, the same current must also flow through the $c x$ edge; thus,

$$
\mathcal{J}_{c x}^{s s}=e^{-B_{c x}}\left(e^{E_{x}} p_{x}^{s s}-e^{E_{c}} p_{c}^{s s}\right)=\mathcal{J}^{s s} .
$$

Given $p_{c}^{s s}, p_{x}^{s s}$, and $\mathcal{J}^{s s}$, we can choose $E_{x}$ such that the sign of $e^{E_{x}} p_{x}^{s s}-e^{E_{c}} p_{c}^{s s}$ is the same as the sign of $\mathcal{J}^{s s}$, and once $E_{x}$ is chosen, we can set $B_{x c}$ to be

$$
B_{x c}=-\ln \frac{\mathcal{J}^{s s}}{e^{E_{c}} p_{c}^{s s}-e^{E_{x}} p_{x}^{s s}} .
$$

Next, we can set the affinity $\phi$ such that the current in the $x w$ edge is in the same direction as in the $c w$ edge: Using

$$
\mathcal{J}_{w x}^{s s}=e^{-B_{w x}}\left(e^{E_{x}-\frac{1}{2} \phi} p_{x}^{s s}-e^{E_{w}+\frac{1}{2} \phi} p_{w}^{s s}\right)=\mathcal{J}^{s s},
$$

we can first choose $\phi$ such that the sign of $e^{E_{x}+\frac{1}{2} \phi} p_{x}^{s s}-$ $e^{E_{w}-\frac{1}{2} \phi} p_{w}^{s s}$ agrees with the sign of $\mathcal{J}^{s s}$, and then calculate $B_{w x}$ such that the above equation holds.

So far, we have seen that by choosing the energy $E_{x}$, the barriers $B_{c x}, B_{w x}$, and the affinity $\phi$, it is possible to "tune" 
the system to have any steady-state distribution. However, once the steady-state distribution is chosen, the current is set by Eq. (46) and cannot be changed. This proves that the constraints indeed limit the system; without them, any $\mathcal{J}^{s s}$ can be achieved with any $\vec{p}^{s s}$ by tuning $E_{w}, E_{c}$, and $B_{c w}$ in Eq. (46).

Next, let us consider the cost of kinetic proofreading, namely, the total entropy production rate: It has three contributions from the three edges. The currents in all three edges are identical and are set by the choice of steady-state probabilities; thus, the differences between the entropy production rates in the different edges are only due to the differences in the $\ln \left(\mathcal{R}_{i j} p_{j}^{s s} / \mathcal{R}_{j i} p_{i}^{s s}\right)$ factors. Any (positive) value of $\sigma_{c x}^{s s}$ can be achieved by choosing the energy of the auxiliary state to be

$$
E_{x}=E_{c}+\frac{\sigma_{c x}^{s s}}{\mathcal{J}^{s s}}+\ln \frac{p_{c}^{s s}}{p_{x}^{s s}},
$$

as can be verified by substituting the above $E_{x}$ in the definition of the entropy production rate. Similarly, it is possible to achieve any positive value of $\sigma_{w x}^{s s}$ by choosing the affinity to be

$$
\phi=E_{x}-E_{w}-\frac{\sigma_{w x}^{s s}}{\mathcal{J}^{s s}}+\ln \frac{p_{x}^{s s}}{p_{w}^{s s}} .
$$

The last contribution to the total entropy production comes from the $c w$ edge and is given by

$$
\sigma_{c w}^{s s}=\mathcal{J}_{s s}\left(E_{c}-E_{w}+\ln D_{\mathrm{NESS}}\right),
$$

where

$$
D_{\mathrm{NESS}}=\frac{p_{c}^{s s}}{p_{w}^{s s}}
$$

is the NESS discrimination ratio. In contrast to the other two edges, this entropy production rate is set by the steadystate distribution and the given state's energies; hence, it cannot be controlled at all. It is therefore a minimum bound on the entropy production rate for any NESS kinetic proofreading to achieve the target probabilities in this system. If $D_{\mathrm{NESS}}=D_{\mathrm{eq}}$, then $\ln D_{\mathrm{NESS}}=E_{w}-E_{c}$; hence, the minimal entropy production is zero, as expected.

In this simple three-state system, any discrimination ratio $D_{\mathrm{NESS}}$ can, in principle, be achieved with an arbitrary low cost in terms of the total entropy production rate, by decreasing both $\overline{p_{c}^{s s}}$ and $\overline{p_{w}^{s s}}$ while keeping their ratio fixed. The price for having low entropy production, however, is that most of the time, the system is neither in the correct state $c$ nor in the incorrect state $w$, but in the auxiliary state $x$. It is possible to achieve $\overline{p_{c}^{s s}}$ arbitrarily close to 1 , but with a logarithmic price in the discrimination ratio $D_{\mathrm{NESS}}$ (even if $\overline{p_{c}^{s s}}=1, \overline{\mathcal{J}^{p s}}$ is finite). This trade-off is common in NESS kinetic proofreading; see, e.g., Refs. [41,42].

Increasing the discrimination ratio between two possible outcomes beyond its equilibrium value might have other applications, for example, in self-assembly. Many selfassembly schemes are based on free-energy minimization, where the ratio of success is dictated by the equilibrium distribution. Controlling this ratio, either in NESS or through periodic driving, might enable self-assembly of structures that cannot be achieved in equilibrium [43].

\section{B. Kinetic proofreading by periodic driving \\ 1. Constraints on periodic driving for kinetic proofreading}

Can kinetic proofreading be achieved without breaking detailed balance but through periodic driving? As we have seen, any NESS system that describes kinetic proofreading can, in principle, be mapped into a SP that mimics the NESS. However, in the construction suggested in Sec. V, $E_{c}$ and $E_{w}$ are not constant as they change throughout the cycle. This makes the construction unsuitable for kinetic proofreading: A periodic driving kinetic proofreading is a time-dependent detailed-balance matrix $\mathcal{W}(t)$, with the additional constraints

$$
\dot{E}_{c}(t)=0, \quad \dot{E}_{w}(t)=0, \quad \dot{B}_{c w}(t)=0 .
$$

Without the constraints on $E_{c}$ and $E_{w}$, it is possible to directly change the equilibrium discrimination ratio, and thus, there is no need for kinetic proofreading. The constraint on $B_{c w}$ is motivated by the physical argument that if the two states $c$ and $w$ are not affected by the external manipulation, the barrier between them should not be affected either. Note that these constraints do not mean that the $\pi_{c}$ and $\pi_{w}$ are time independent since they are also a function of the time-dependent $E_{x}(t)$ through the partition function $Z$. However, their ratio,

$$
\frac{\pi_{c}(t)}{\pi_{w}(t)}=\delta
$$

is time independent. The only physical parameters in $\mathcal{W}(t)$ that can be manipulated are those of the auxiliary state $x$, namely, its energy $E_{x}$ and its barrier heights, $B_{w x}$ and $B_{c x}$. Mapping between the kinetic proofreading NESS to a constrained $\mathcal{W}(t)$ such that Eq. (13) holds shows that the effect of any external drive $\phi$ can be exactly mimicked by a time-dependent auxiliary state.

As in the NESS kinetic proofreading, once the target averaged probabilities $\overline{p_{i}^{p s}}$ are chosen, $\overline{\mathcal{J}^{p s}}$ is set by the constraints in Eq. (53): 


$$
\begin{aligned}
\overline{\mathcal{J}_{c w}^{p s}} & =\int_{0}^{T}\left(\mathcal{W}_{c w}(t) p_{w}^{p s}(t)-\mathcal{W}_{w c}(t) p_{c}^{p s}(t)\right) d t \\
& =\mathcal{W}_{c w} \int_{0}^{T} p_{w}^{p s}(t) d t-\mathcal{W}_{w c} \int_{0}^{T} p_{c}^{p s}(t) d t \\
& =e^{-B_{c w}}\left(e^{E_{w}} \overline{p_{w}^{p s}}-e^{E_{c}} \overline{p_{c}^{p s}}\right) .
\end{aligned}
$$

Comparing with Eq. (46), we note that $\overline{p_{i}^{p s}}=p_{i}^{s s}$ implies $\overline{\mathcal{J}_{c w}^{p s}}=\mathcal{J}_{c w}^{s s}$. In other words, the choice of distribution, $p_{i}^{s s}$ or $\overline{p_{i}^{p s}}$, uniquely defines the corresponding currents, $\mathcal{J}_{c w}^{s s}$ or $\overline{\mathcal{J}_{c w}^{p s}}$, and a given set of probabilities $p_{i}^{s s}=\overline{p_{i}^{p s}}$ gives rise to the same currents in both the periodic and steady-state systems.

In what follows, we construct a periodic detailed balance rate matrix $\mathcal{W}(t)$ with a given $E_{c}, E_{w}$, and $B_{c w}$, such that its time averaged probabilities can be any (normalized) probability vector $\overline{p_{i}^{p s}}$, using the tools developed in the previous sections. For simplicity, we first show how to construct the protocol without considering the entropy production rates. Then, we show that the entropy production rates can be controlled by tuning the free parameters in the construction and that the bound on the minimal averaged total entropy production rate is equal to the bound in NESS kinetic proofreading.

\section{Construction of a proofreading protocol}

We start with a simple, physical intuition for the kinetic proofreading protocol. As evident from Eq. (55), the energies of the states $c$ and $w$, together with the barrier between them, as well as the target averaged probabilities, dictate the averaged current that flows between these states. Without loss of generality, we assume that $\delta<1$ but $\overline{p_{c}^{p s}}>$ $\overline{p_{w}^{p s}}$ (the other cases can be worked out similarly). Under this assumption, $\overline{\mathcal{J}_{w c}^{p s}}>0$; namely, on average, probability flows along the $w c$ edge from the state $c$ into the state $w$. However, because of the no-current-loops condition, it is not possible to compensate for both the probability that flows out of $c$ and the probability that flows into $w$ at each instance of time since the former requires current from $x$ into $c$ and the latter from $w$ into $x$, which, together with the current on the $w c$ edge, form a current loop. Thus, as in Sec. V, we divide the cycle time into two halves. In the first half-cycle, the probability current $\mathcal{J}_{c x}^{a}$ that flows into the state $c$ and which is the only probability current flowing into the state $c$ over the entire cycle must compensate for all the probability currents that flow out of the state $c$ during a complete cycle, namely, for $\mathcal{J}_{w c}^{a}, \mathcal{J}_{w c}^{a}$, and $\mathcal{J}_{x c}^{a}$ (see Fig. 6 for the current pattern in the two halves of the cycle). By the no-loop condition, during the same half-cycle, the current along the $x w$ edge must flow from $x$ into $w$, further increasing the accumulated probability in $w$. These currents can be achieved by increasing the energy of the auxiliary state, lowering the barrier between $x$ and $c$ and increasing

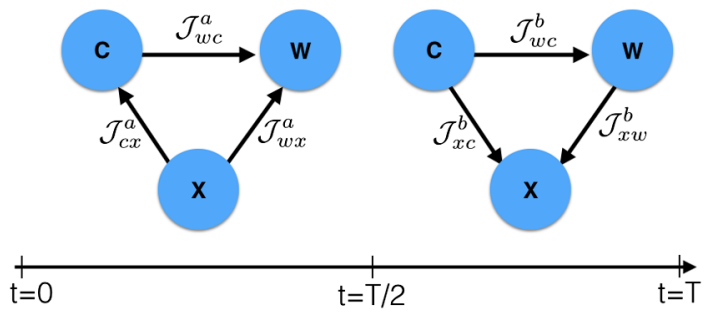

FIG. 6. Patters of currents in periodically driven kinetic proofreading. In the first half-cycle (left), both currents are out of the state $x$, and in the second half-cycle (right), they are both into the state $x$.

the barrier between $x$ and $w$, to ensure that most of the probability from $x$ flows into $c$ and not $w$. In the second half-cycle, the probability that accumulates during a cycle in $w$ is evacuated into $x$. To avoid a current loop, in this half-cycle some of the probability from $c$ must also backflow into $x$. This is achieved by lowering the energy of $x$, lowering the barrier between $w$ and $x$, and raising the barrier between $x$ and $c$, to ensure that most of the probability that flows into $x$ comes from $w$, rather than from $c$.

To make this intuitive heuristic into a quantitative protocol, we apply the tools developed so far. This is done in three steps. In the first step, we choose the master equation's solution, $\vec{p}^{p s}(t)$, such that $\overline{p_{i}^{p s}}$ are the target probabilities. In the second step, we set $E_{x}$ in the two halfperiods such that the current pattern is consistent with the no-current-loops condition. In the last step, we use the transformation of the symmetric part of the rate matrix (described in Sec. IV C) to set $B_{w x}(t)$ and $B_{c x}(t)$ such that $\vec{p}^{p s}(t)$ is the solution of the master equation. These steps are detailed below.

Step 1: Choosing the solution $\vec{p}(t)$.- To construct $\mathcal{W}(t)$, we first set $p_{i}^{p s}(t)$ to be continuous and piecewise-linear in time, with opposite slopes in the two half-cycles and with the target averaged probabilities

$$
\begin{aligned}
& p_{c}^{p s}(t)= \begin{cases}\overline{p_{c}^{p s}}-\frac{m T}{4}+m t & 0 \leq t \leq \frac{T}{2} \\
\overline{p_{c}^{p s}}+\frac{3 m T}{4}-m t & \frac{T}{2}<t \leq T,\end{cases} \\
& p_{w}^{p s}(t)= \begin{cases}\overline{p_{w}^{p s}}-\frac{m T}{4}+m t & 0 \leq t \leq \frac{T}{2} \\
\overline{p_{w}^{p s}}+\frac{3 m T}{4}-m t & \frac{T}{2}<t \leq T .\end{cases}
\end{aligned}
$$

The $m T / 4$ and $3 m T / 4$ constants assure that the average probabilities are indeed $\overline{p_{c}^{p s}}$ and $\overline{p_{w}^{p s}}$. Note that the slopes of $p_{c}^{p s}(t)$ and $p_{w}^{p s}(t)$ are equal to each other but are opposite in sign to the slope of $p_{x}^{p s}(t)=1-p_{c}^{p t}(t)-p_{w}^{p s}(t)$, which is $2 m$ in amplitude. The slope $m$ is chosen subject to the following constraint: 


$$
m>e^{-B_{c w}} \max _{0 \leq t \leq T}\left|\frac{p_{w}^{p s}(t)}{e^{-E_{w}}}-\frac{p_{c}^{p s}(t)}{e^{-E_{c}}}\right|,
$$

where $T>0$ is chosen arbitrarily. Once $m$ is chosen, we have to check that all the probabilities are bounded away from 1 and 0 . If they are not, we rechoose $T$ to be short enough such that they become bounded away from 0 and 1 , and update $\vec{p}_{w}^{p s}(t)$ and $\vec{p}_{w}^{p s}(t)$, but not $m$, accordingly. The condition on $m$ ensures that the magnitude of the current between states $c$ and $w$, given by

$$
\left|\mathcal{J}_{c w}^{p s}(t)\right|=e^{-B_{c w}}\left|\frac{p_{w}^{p s}(t)}{e^{-E_{w}}}-\frac{p_{c}^{p s}(t)}{e^{-E_{c}}}\right|,
$$

is smaller than the magnitudes $\dot{p}_{c}^{p s}=\dot{p}_{w}^{p s}= \pm m$ at all $t$. Since $\dot{p}_{c}^{p s}=\mathcal{J}_{c x}^{p s}+\mathcal{J}_{c w}^{p s}$ and similarly $\dot{p}_{w}^{p s}=\mathcal{J}_{w x}^{p s}+\mathcal{J}_{w c}^{p s}$, the signs of $\mathcal{J}_{c x}$ and $\mathcal{J}_{w x}$ are always identical to the signs of $\dot{p}_{c}^{p s}$ and $\dot{p}_{w}^{p s}$, and hence to each other. Therefore, $\mathcal{J}_{c x}^{p s}$ and $\mathcal{J}_{w x}^{p s}$ are either both into the auxiliary state $x$ or are both out of it, and there are no current loops.

The condition on $m$ can also be viewed as a condition that assures that the dynamics is far from the adiabatic limit: If the changes in $\vec{p}$ are slow enough such that the dynamics follows the adiabatic approximation, then $\vec{p}^{p s}(t) \approx \vec{\pi}(t)$; thus, $D \approx D_{e q}$ and there is no kinetic proofreading.

Step 2: Setting $E_{x}(t)$.- - In the previous step, the values of $\dot{\vec{p}}(t)$, and therefore of the currents in the system, were chosen. This was done consistently with the no-currentloops condition. Next, we choose the auxiliary state energy in the two halves of the cycle, $E_{x}^{a}$ and $E_{x}^{b}$ (as before, superscripts $a$ and $b$ stand for the first and second halfcycles, respectively) in a way that is consistent with the directions of the currents. To this end, we can express the periodic state current between the $i$ th and $j$ th states as

$$
\mathcal{J}_{i j}^{p s}(t)=e^{-B_{i j}(t)}\left(\frac{p_{j}^{p s}(t)}{e^{-E_{j}(t)}}-\frac{p_{i}^{p s}(t)}{e^{-E_{i}(t)}}\right) .
$$

The direction of the current $\mathcal{J}_{i j}^{p s}(t)$ is thus given by the sign of $\left\{\left[p_{j}^{p s}(t)\right] /\left[e^{-E_{j}(t)}\right]\right\}-\left\{\left[p_{i}^{p s}(t)\right] /\left[e^{-E_{i}(t)}\right]\right\}$. To ensure that in the first half-cycle the directions of the currents are indeed from the auxiliary state $x$ into states $c$ and $w$, we therefore must choose $E_{x}^{a}$ such that both

$$
\frac{p_{x}^{p s}(t)}{e^{-E_{x}^{a}}}-\frac{p_{c}^{p s}(t)}{e^{-E_{c}}}>0
$$

and

$$
\frac{p_{x}^{p s}(t)}{e^{-E_{x}^{a}}}-\frac{p_{w}^{p s}(t)}{e^{-E_{w}}}>0,
$$

which means

$$
\begin{aligned}
& \frac{p_{x}^{p s}(t)}{p_{c}^{p s}(t)}>\frac{e^{-E_{x}^{a}}}{e^{-E_{c}}}=\frac{\pi_{x}^{a}}{\pi_{c}}, \\
& \frac{p_{x}^{p s}(t)}{p_{w}^{p s}(t)}>\frac{e^{-E_{x}^{a}}}{e^{-E_{w}}}=\frac{\pi_{x}^{a}}{\pi_{w}} .
\end{aligned}
$$

By the assumption $\delta<1$ and $p_{c}^{p s}(t)>p_{w}^{p s}(t)$, the second inequality follows from the first one. Using $\pi_{w}=\pi_{c} \delta^{-1}$ [from Eq. (54)] and $\pi_{w}+\pi_{c}+\pi_{x}=1$ (probability normalization), we can express

$$
\pi_{x}=1-\pi_{c}\left(1+\delta^{-1}\right)
$$

Substituting this in the above inequality gives

$$
\frac{p_{x}^{p s}(t)}{p_{c}^{p s}(t)}>\frac{1-\pi_{c}\left(1+\delta^{-1}\right)}{\pi_{c}}=\pi_{c}^{-1}-\left(1+\delta^{-1}\right) .
$$

This gives the exact condition for choosing $\pi_{c}^{a}$ :

$$
\frac{1}{1+\delta^{-1}}>\pi_{c}^{a}>\max _{t} \frac{1}{\frac{p_{x}^{p s}(t)}{p_{c}^{p s}(t)}+1+\delta^{-1}},
$$

where the left inequality follows from the equality $\pi_{c}^{p s}(t)=$ $\delta^{-1} \pi_{w}^{p s}(t)$ and probability normalization. From $\pi_{c}^{a}$ (and similarly for $\pi_{c}^{b}$ ), we can solve for $E_{x}^{a}$ (for $E_{x}^{b}$ ). Using

$$
\frac{e^{-E_{c}}}{e^{-E_{x}^{a, b}}+e^{-E_{c}}+e^{-E_{w}}}=\pi_{c}^{a, b},
$$

we can extract

$$
E_{x}^{a, b}=-\ln \left(e^{-E_{c}}\left(\frac{1}{\pi_{c}^{a, b}}-1\right)-e^{-E_{w}}\right)
$$

A similar argument can be applied to the second half-cycle. In this case, the currents should be from states $c$ and $w$ into the state $x$; thus,

$$
\begin{aligned}
& \frac{p_{x}^{p s}(t)}{e^{-E_{x}^{b}}}-\frac{p_{c}^{p s}(t)}{e^{-E_{c}}}<0, \\
& \frac{p_{x}^{p s}(t)}{e^{-E_{x}^{b}}}-\frac{p_{w}^{p s}(t)}{e^{-E_{w}}}<0 .
\end{aligned}
$$

By the assumption $\delta<1$ and $p_{c}^{p s}(t)>p_{w}^{p s}(t)$, the first inequality follows from the second one, which can be written as

$$
\frac{p_{x}^{p s}(t)}{p_{w}^{p s}(t)}<\frac{e^{-E_{x}^{b}}}{e^{-E_{w}}}=\frac{\pi_{x}^{b}}{\pi_{w}}=\frac{1-\pi_{c}\left(1+\delta^{-1}\right)}{\delta^{-1} \pi_{c}},
$$

namely, 


$$
\pi_{c}^{b}<\min _{t} \frac{1}{1+\delta^{-1}\left(1+\frac{p_{x}^{p s}(t)}{p_{w}^{p s}(t)}\right)}
$$

Once $\pi_{c}^{b}$ is chosen, $E_{x}^{b}$ can be expressed through Eq. (68).

Note that both $E_{c}^{a}$ and $E_{c}^{b}$ are not uniquely set but can be chosen in some range of values. Although different choices lead to the same averaged probabilities and current, their averaged entropy production rates are different.

Step 3: Transforming the symmetric part of the rate matrix.-So far, the instantaneous equilibrium distribution $\vec{\pi}(t)$ and the periodic solution $\vec{p}^{p s}(t)$ were chosen such that there are no current loops. It is therefore possible to use the transformation of the symmetric part of the rate matrix, discussed in Sec. IV C, to adjust the magnitudes of the currents $\mathcal{J}_{c x}^{p s}(t)$ and $\mathcal{J}_{w x}^{p s}(t)$ to agree with $\dot{\vec{p}}$. In the first halfcycle,

$$
\begin{aligned}
& \mathcal{J}_{c x}^{p s}=\dot{p}_{c}-\mathcal{J}_{c w}^{p s}=m+e^{-B_{c w}}\left(\frac{p_{c}^{p s}}{e^{-E_{c}}}-\frac{p_{w}^{p s}}{e^{-E_{w}}}\right), \\
& \mathcal{J}_{w x}^{p s}=\dot{p}_{w}-\mathcal{J}_{w c}^{p s}=m-e^{-B_{c w}}\left(\frac{p_{c}^{p s}}{e^{-E_{c}}}-\frac{p_{w}^{p s}}{e^{-E_{w}}}\right),
\end{aligned}
$$

where the explicit time dependence is omitted. The condition on $m$ in Eq. (58) ensures that both of these currents are indeed positive. Moreover, by the current definition,

$$
\begin{aligned}
& \mathcal{J}_{c x}^{p s}(t)=e^{-B_{x c}(t)}\left(\frac{p_{c}^{p s}(t)}{e^{-E_{c}}}-\frac{p_{x}^{p s}(t)}{e^{-E_{x}^{a}}}\right), \\
& \mathcal{J}_{w x}^{p s}(t)=e^{-B_{x w}(t)}\left(\frac{p_{w}^{p s}(t)}{e^{-E_{w}}}-\frac{p_{x}^{p s}(t)}{e^{-E_{x}^{a}}}\right) .
\end{aligned}
$$

Combining Eq. (75) with Eq. (73), it is straightforward to solve for $B_{x c}(t)$, and similarly from Eq. (76)) with Eq. (74) to solve for $B_{x w}(t)$. The second half-cycle values of $B_{x w}$ and $B_{x c}$ can be solved in the same way. As expected from the heuristic description, in the first half-cycle, $B_{x c}(t)<B_{x w}(t)$ and in the second half-cycle, $B_{x c}(t)>B_{x w}(t)$. Using these $B_{c x}(t)$ and $B_{w x}(t)$ together with $E_{x}^{a}$ in the first half-cycle and $E_{x}^{b}$ in the second half, we get, by construction, the targeted probabilities as the averages of the periodic solution $\vec{p}^{p s}(t)$.

The construction for periodically driven kinetic proofreading protocol given above uses the same tools as the construction of the NESS-SP mapping, with a crucial difference: In the NESS-SP mapping, the symmetric part of the rate is kept fixed during each half-cycle, and the equilibrium distribution changes with time; in the kinetic proofreading protocol, it is exactly the opposite. This difference makes it simpler to construct a protocol that respects the constraint on the equilibrium distribution.

\section{Entropic cost of kinetic proofreading SP}

To complete the NESS-SP equivalence in this three-state system kinetic proofreading example, we should, in principle, match the entropy production rates of each of the edges in the SP protocol to those of the NESS. While this is definitely possible, for simplicity, we limit our discussion only to the total averaged entropy production rate, which is the total cost of the kinetic proofreading protocol. In what follows, we show that the total entropy production is bounded from below and that its minimal value is the same as that of the NESS process. In other words, NESS and SP kinetic proofreading can be achieved at the same entropy production cost. To this end, we first split the total entropy production rate as follows:

$$
\begin{aligned}
\overline{\sigma_{i j}} & =\frac{1}{T} \int_{0}^{T} \mathcal{J}_{i j}(t) \ln \frac{\mathcal{W}_{i j}(t) p_{j}^{p s}(t)}{\mathcal{W}_{j i}(t) p_{i}^{p s}(t)} d t \\
& =\frac{1}{T} \int_{0}^{T}\left(\mathcal{J}_{i j}(t) \ln \frac{\mathcal{W}_{i j}(t)}{\mathcal{W}_{j i}(t)}+\mathcal{J}_{i j}(t) \ln \frac{p_{j}^{p s}(t)}{p_{i}^{p s}(t)}\right) d t .
\end{aligned}
$$

The first term, $\int_{0}^{T} \mathcal{J}_{i j}(t) \ln \left\{\left[\mathcal{W}_{i j}(t)\right] /\left[\mathcal{W}_{j i}(t)\right]\right\} d t$, is commonly associated with the entropy production of the heat bath to which the system is coupled, and the second term, $\int_{0}^{T} \mathcal{J}_{i j}(t) \ln \left\{\left[p_{j}^{p s}(t)\right] /\left[p_{i}^{p s}(t)\right]\right\} d t$, to the entropy production of the system itself. For the periodic state, the sum of all the latter vanishes [37],

$$
\sum_{i j} \int_{0}^{T} \mathcal{J}_{i j}(t) \ln \frac{p_{j}^{p s}(t)}{p_{i}^{p s}(t)} d t=0
$$

Thus, the total entropy production rate is given by

$$
\begin{aligned}
\sum_{i<j} \overline{\sigma_{i j}} & =\frac{1}{T} \sum_{i<j} \int_{0}^{T} \mathcal{J}_{i j}(t) \ln \frac{\mathcal{W}_{i j}(t)}{\mathcal{W}_{j i}(t)} d t \\
& =\frac{1}{T} \sum_{i<j} \int_{0}^{T} \mathcal{J}_{i j}(t)\left(E_{j}-E_{i}\right) d t .
\end{aligned}
$$

As the energies are fixed during each half-cycle, this expression can be simplified into

$$
\begin{aligned}
\sum_{i, j} \overline{\sigma_{i j}}= & \overline{\mathcal{J}_{w c}^{p s}}\left(E_{c}-E_{w}\right)+\frac{1}{2} \overline{\mathcal{J}_{w x}^{a}}\left(E_{x}^{a}-E_{w}\right) \\
& +\frac{1}{2} \overline{\mathcal{J}_{c x}^{a}}\left(E_{x}^{a}-E_{c}\right)+\frac{1}{2} \overline{\mathcal{J}_{x w}^{b}}\left(E_{w}-E_{x}^{b}\right) \\
& +\frac{1}{2} \overline{\mathcal{J}_{x c}^{b}}\left(E_{c}-E_{x}^{b}\right) \\
= & m\left(E_{x}^{a}-E_{x}^{b}\right),
\end{aligned}
$$

where we used $\overline{\mathcal{J}_{x w}^{b}}-\overline{\mathcal{J}_{w x}^{a}}=2 \overline{\mathcal{J}_{x w}^{p s}}, \overline{\mathcal{J}_{x c}^{b}}-\overline{\mathcal{J}_{c x}^{a}}=-2 \overline{\mathcal{J}_{c x}^{p s}}$, and as there is only one averaged current in the system, 
$\overline{\mathcal{J}_{x w}^{p s}}=\overline{\mathcal{J}_{w c}^{p s}}=\overline{\mathcal{J}_{c x}^{p s}}$ as well. In addition, we used $\mathcal{J}_{w x}^{a}+$ $\mathcal{J}_{c x}^{a}=-\dot{p}_{x}^{a}=2 m$ and similarly for the second halfcycle, $\mathcal{J}_{x w}^{b}+\mathcal{J}_{x c}^{b}=\dot{p}_{x}^{b}=2 m$.

We can now bound the minimal total entropy production rate required to achieve a given SP discrimination factor, $D_{\mathrm{SP}}$, defined by

$$
D_{\mathrm{SP}}=\overline{p_{c}^{p s}} / \overline{p_{w}^{p s}}
$$

using the above protocol. We first note that the constraint on $m$ in Eq. (58) implies that $m \geq \overline{\mathcal{J}_{w c}^{p s}}$, where the equality is achieved only in the limit $T \rightarrow 0$. In addition, we can bound $E_{x}^{a}$ and $E_{x}^{b}$, using Eqs. (66), (68), and (72):

$$
\begin{gathered}
E_{x}^{a}>-\ln \left(e^{-E_{c}}\left(\delta^{-1}+\min _{t} \frac{p_{x}^{p s}(t)}{p_{c}^{p s}(t)}\right)-e^{-E_{w}}\right), \\
E_{x}^{b}<-\ln \left(e^{-E_{c}} \delta^{-1}\left(1+\max _{t} \frac{p_{x}^{p s}(t)}{p_{w}^{p s}(t)}\right)-e^{-E_{w}}\right),
\end{gathered}
$$

but $e^{-E_{c}} \delta^{-1}=e^{-E_{w}} ;$ thus,

$$
\begin{aligned}
& E_{x}^{a}>E_{c}-\ln \left(\min _{t} \frac{p_{x}^{p s}(t)}{p_{c}^{p s}(t)}\right), \\
& E_{x}^{b}<E_{w}-\ln \left(\max _{t} \frac{p_{x}^{p s}(t)}{p_{w}^{p s}(t)}\right) .
\end{aligned}
$$

Substituting these bounds into the total entropy production rate Eq. (80) and using

$$
\max _{t} \frac{p_{c}^{p s}(t)}{p_{w}^{p s}(t)} \geq D_{S P}
$$

we get

$$
\bar{\sigma} \geq \overline{\mathcal{J}_{w c}^{p s}}\left(E_{c}-E_{w}+\ln D_{\mathrm{SP}}\right) .
$$

This bound, which can be attained in the $T \rightarrow 0$ limit by a proper choice of $m, E_{x}^{a}$ and $E_{x}^{b}$, is exactly the bound on NESS kinetic proofreading (for the same target probabilities) given in Eq. (51). On the other hand, the total averaged entropy production rate can be made arbitrarily large by choosing $m$ to be large enough. This shows that any NESS kinetic proofreading in this system, namely, any value of $\phi, E_{x}, B_{x w}$, and $B_{x c}$, can be mimicked (in terms of probabilities, current, and total entropy production rate) by the protocol described above.

\section{CONCLUSIONS AND FUTURE PERSPECTIVE}

In this paper, we have shown that the NESS and SP paradigms are equivalent in terms of averaged probabilities, currents, and entropy production rates, and we obtained an explicit mapping between them. We then applied these results to construct a SP kinetic-proofreading protocol in a three-state system. These results are the first steps in clarifying the precise relationship between these two paradigms, and they were established using a powerful and, in our view, somewhat surprising observation: Although finding the average properties of an arbitrary SP protocol is a difficult problem that, in general, can only be solved numerically, the inverse problem-finding a time-periodic protocol that has a desired set of averaged properties-is an easy problem which only requires solving a set of algebraic linear equations.

The mapping between NESS and SP presented above was not intended as a set of operational instructions for experimental realization: Indeed, in most experimental realizations, not all the rates can be individually controlled; hence, the specific protocol suggested above might not be applicable. Rather, our intention is to show that SP are as versatile a tool as NESS in controlling molecular motors and other stochastic processes and that it is possible to design driving protocols with desired outcomes.

Biological systems suggest inspiring examples of complicated molecular machines with different functionalities - from transport of ingredients by molecular motors to kinetic proofreading in biomolecular synthesis-all operating at NESS. Although it is possible to break detailed balance in the laboratory (e.g., by applying external voltage or creating temperature or concentration gradients), it is difficult to design a molecular machine that, similar to biological molecular machines, exploits external resources to drive the system in exactly the desired way. Expanding the tools at our disposal to include periodic driving might therefore significantly increase our ability to control molecular motors. The toolbox developed in this manuscript suggests "design principles" for periodic driving and shows that tailoring a time-periodic protocol with desired properties is unexpectedly simple.

Many important theoretical and experimental aspects of the mapping were not addressed in the current manuscript. On the theoretical side, these include a comparison of the fluctuations around the averaged values in the two paradigms, the relaxation rates to the steady and periodic states, and the construction of mappings that preserve properties of interest other than the ones we studied. As an example of a potentially important theoretical application, consider the heat, power, and efficiency in heat engines. Some heat engines, e.g., thermo-electrical heat engines, are best modeled as NESS: The different heat baths generate heat fluxes that violate detailed balance. In contrast, reciprocating heat engines (e.g., the Carnot, Otto, or Diesel cycles) are naturally modeled as SP [44]. Mapping the heat, power, and efficiency between these two paradigms (with additional constraints on the temperature) might enable transferring many known relations in NESS heat engines (e.g., efficiency at maximal power [45-47]) to SP heat engines, where such general relations have not been proven yet.

It will be interesting to extend our analysis to other cases as well: One natural example is diffusive (rather than discrete-state) stochastic dynamics. Diffusive systems evolving in time-periodic potentials can act as stochastic 
pumps [48], generating nonvanishing average currents, provided they satisfy the conditions of a no-pumping theorem $[22,49]$ analogous to that for discrete-state systems [20-24]. These similarities suggest that periodically driven diffusive systems might be able to mimic diffusive nonequilibrium steady states, and vice versa. A different possible extension is to find an analogous mapping for open quantum systems, where in addition to amplitudes, phases are of importance.

There are a number of experimentally motivated questions for which the tools we developed might be useful. Examples include the following: (i) What are the exact constraints under which the equivalence still holds? In kinetic proofreading, we have provided a specific example where the equivalence holds even with partial control over the system. However, the minimal degree of control required to achieve equivalence remains an open question. (ii) What are the attainable values of various properties (e.g., averaged currents or probability ratios) under a given set of experimental constraints? For example, consider the case in which only the surrounding temperature can be controlled: Can significant changes in the ratio of probabilities be achieved by periodically changing the temperature only? (iii) In some experiments, it might be possible both to break detailed balance and to drive the system periodically. Finding protocols that optimize properties of interest under specific experimental constraints then becomes a natural question.

\section{ACKNOWLEDGMENTS}

C. J. and Y. S. acknowledge financial support from the U.S. Army Research Office under Contract No. W911NF13-1-0390. O. R. acknowledges financial support from the James S. McDonnell Foundation.

\section{APPENDIX A: DETAILS OF THE SPECIFIC EXAMPLE}

The example we consider is shown in Fig. 2. It has four states with $\vec{p}^{s s}=(0.1,0.2,0.3,0.4)$. The spanning-tree edges are the dashed-dotted lines (red), and the fundamental current edges are the solid line lines (black). The fundamental currents were chosen such that they form a loop, and their values are $\mathcal{J}_{31}^{s s}=3, \mathcal{J}_{43}^{s s}=2$, and $\mathcal{J}_{14}^{s s}=1$. Once these currents are chosen, the currents for the spanning-tree edges are dictated by Kirchoff's law-the sum of currents in each vertex must be zero. The corresponding current matrix is

$$
\mathcal{J}^{s s}=\left(\begin{array}{cccc}
0 & 2 & -3 & 1 \\
-2 & 0 & 1 & 1 \\
3 & -1 & 0 & -2 \\
-1 & -1 & 2 & 0
\end{array}\right) .
$$

To set $\mathcal{S}$, we choose the entropy production rate to be 1 along all the edges. Using Eq. (16), the matrix $\mathcal{S}$ is given in this case by

$$
\mathcal{S}=\left(\begin{array}{cccc}
-14.248 & 4.083 & 9.0832 & 1.082 \\
4.083 & -6.2469 & 1.082 & 1.082 \\
9.0832 & 1.0820 & -14.2481 & 4.083 \\
1.082 & 1.082 & 4.083 & -6.2469
\end{array}\right)
$$

and using Eq. (14), $\mathcal{R}$ can be calculated, though we will not need it in what follows.

Let us demonstrate our construction on this NESS. For the first step, we choose

$$
\tilde{\mathcal{S}}=\left(\begin{array}{cccc}
-3 & 1 & 1 & 1 \\
1 & -3 & 1 & 1 \\
1 & 1 & -3 & 1 \\
1 & 1 & 1 & -3
\end{array}\right), \quad \vec{\pi}^{a}=\left(\begin{array}{c}
\frac{1}{4} \\
\frac{1}{4} \\
\frac{1}{4} \\
\frac{1}{4}
\end{array}\right) .
$$

We next note that $\min _{i j}\left[\log \mathcal{R}_{i j} p_{j}^{s s}-\log \mathcal{R}_{j i} p_{i}^{s s}\right]=1 / 3$. If we therefore choose $\vec{q}^{a}$ such that $\left|\log \pi_{i}^{a}-\log q_{i}^{a}\right|<1 / 6$, condition (25) is automatically satisfied. Any vector close enough to $\vec{\pi}^{a}$ will do. As an example, we use $\vec{q}^{a}=(0.23,0.24,0.26,0.27)$. In the second half of the period, these correspond to $\vec{\pi}^{b}=(4,4,4,4)$ and $\vec{q}^{b}=$ (4.3478, 4.1667, 3.8462, 3.7037).

The next step requires the signs of the currents generated by $\tilde{\mathcal{R}}^{a}=\tilde{\mathcal{S}}\left(\Pi^{a}\right)^{-1}$ with the probability $\vec{q}^{a}$ :

$\operatorname{sign}\left(\tilde{\mathcal{J}}_{13}^{1}\right)=+1, \quad \operatorname{sign}\left(\tilde{\mathcal{J}}_{14}^{1}\right)=+1, \quad \operatorname{sign}\left(\tilde{\mathcal{J}}_{34}^{1}\right)=+1$.

Note that the direction of the current in the 1-4 edge is the same as the desired current orientation, but for the other two edges, the directions are different from the desired currents.

In the second step, we solve Eq. (32) for $\alpha_{i j}$, which gives

$$
\begin{aligned}
\alpha_{13} & =1.7188, \\
\alpha_{14} & =5.2366, \\
\alpha_{34} & =12.2484 .
\end{aligned}
$$

Together with the directions, the fundamental currents in the two half-cycles are given by

$$
\begin{array}{r}
\mathcal{J}_{13}^{1}=5.156, \quad \mathcal{J}_{14}^{1}=7.2366, \\
\mathcal{J}_{34}^{1}=24.4969, \quad \mathcal{J}_{13}^{2}=-11.156, \\
\mathcal{J}_{14}^{2}=-5.2366, \quad \mathcal{J}_{34}^{2}=-28.4969 .
\end{array}
$$

Note that the averages are the same as the desired values. 
In the third step, we solve for the spanning-tree currents, by solving Eqs. (34) and (35) for $\mathcal{J}_{12}^{1,2}, \mathcal{J}_{23}^{1,2}$, and $\mathcal{J}_{24}^{1,2}$. They are given by

$$
\begin{array}{cr}
\mathcal{J}_{12}^{1}=-25.4965, & \mathcal{J}_{23}^{1}=13.4933, \\
\mathcal{J}_{24}^{1}=9.4902, & \mathcal{J}_{12}^{2}=-21.4965, \\
\mathcal{J}_{23}^{2}=-11.4933, & \mathcal{J}_{24}^{2}=-7.4902 .
\end{array}
$$

In the fourth step, we calculate $\mathcal{S}^{a}$ and $\mathcal{S}^{b}$ using Eq. (39). Here, we present only the upper part as they are symmetric:

$$
\begin{aligned}
\mathcal{S}^{a} & =\left(\begin{array}{cccc}
-725.6 & 637.4 & 43 & 45.2 \\
& -885.2 & 168.7 & 79.1 \\
& -842.1 & & 612.4 \\
& & & -736.7
\end{array}\right), \\
\mathcal{S}^{b} & =\left(\begin{array}{cccc}
-596.1 & 474.6 & 89 & 32.5 \\
& -682.8 & 143.4 & 64.7 \\
& & -1032.6 & 800.2 \\
& & & -897.4
\end{array}\right) .
\end{aligned}
$$

In the last step, we first calculate $\partial_{t} \vec{p}$ on the two halfcycles by proper summation of the currents: $\partial_{t} \vec{p}^{a}=$ $(37.89,-2.51,5.85,-41.22)$ and $\partial_{t} \vec{p}^{b}=(-37.89,2.51$, $-5.85,41.22)$, which, as expected, cancel each other. These are the slopes of the $p(t)$ 's in the first and second halves of the period. To keep $0<p(t)<1$, we need to choose $T$ small enough, say, for simplicity, $T=0.01$. Using $T$, we can calculate $\vec{p}^{a, b}(t)$, which are the actual probability distributions in the two halves. These linear functions are plotted in Fig. 4. Plugging these into Eq. (40) gives $\mathcal{W}(t)$.

\section{APPENDIX B: ZERO CURRENTS IN THE NESS}

If $\mathcal{J}_{i j}^{s s}=0$ for some edges, then clearly $\sigma_{i j}^{s S}=0$ for the same edges as well. However, since $\sigma_{i j}^{p s}(t) \geq 0$ identically [this follows from Eqs. (10) and (11)], we must have $\sigma_{i j}^{p s}(t)=0$ for all $t$ in order for the time-averaged entropy production rate of the periodic state to be zero. This can only happen if the currents along these edges are zero at all times, $\mathcal{J}_{i j}^{p s}(t)=0$. A simple prescription to set these currents to zero is to use the following modified rate matrix:

$$
\tilde{\mathcal{R}}_{i j}=\left(\tilde{\mathcal{S}}+\frac{3}{4} \mathcal{J}^{s s}\right) \mathcal{P}^{-1}
$$

where $\mathcal{P}$ is the matrix with the steady state of $\mathcal{R}$ on its diagonal, $\mathcal{J}^{s s}$ is the steady-state current matrix of $\mathcal{R}$ (but with a different factor in front of it), and

$$
\tilde{\mathcal{S}}_{i j}= \begin{cases}0 & \mathcal{J}_{i j}=0 \text { and } i \neq j \\ \frac{3}{2} \mathcal{S}_{i j} & \mathcal{J}_{i j} \neq 0\end{cases}
$$

with the diagonal elements of $\tilde{\mathcal{S}}$ changed to make the sum of columns zero. This modified rate matrix, in which the edges with zero steady-state currents have been "removed" ( $\tilde{\mathcal{R}}_{i j}=0$ on these edges), has the same steady state as $\mathcal{R}$, but its steady-state currents are 1.5 times larger than those of $\mathcal{R}$. From Eq. (16), it also follows that $\tilde{\sigma}_{i j}^{s s}=1.5 \sigma_{i j}^{s s}$.

Note that forcing some of the $\tilde{\mathcal{R}}_{i j}$ to be zero might make the time-dependent system nonergodic since it might disconnect some of the states from the others at all times. This, however, can be overcome by dividing the time interval into three equal intervals rather than two. In the first two parts, we repeat the construction as before, but using $\tilde{\mathcal{R}}$ instead of $\mathcal{R}$. In the last interval, we choose $\mathcal{W}(t)=\mathcal{S}^{c}\left(\Pi^{c}\right)^{-1}$, with $\mathcal{S}_{i j}^{c}=1$ for any $i \neq j$ and $\Pi^{c}$ the diagonal matrix with $\vec{p}^{p s}(t=0)$ on its diagonal. With this construction, there are no currents in the periodic solution during the last time interval, the average currents and entropy production rates are the required ones, and the last interval ensures that the system is ergodic.

[1] R. Zwanzig, Nonequilibrium Statistical Mechanics (Oxford University Press, New York, 2001).

[2] C Jarzynski, Equalities and Inequalities: Irreversibility and the Second Law of Thermodynamics at the Nanoscale, Annu. Rev. Condens. Matter Phys. 2, 329 (2011).

[3] E. W. Montroll and K. E. Shuler, Studies in Nonequilibrium Rate Processes. I. The Relaxation of a System of Harmonic Oscillators, J. Chem. Phys. 26, 454 (1957).

[4] H. Ge, H. Qian, and M. Qian, Stochastic Theory of Nonequilibrium Steady States and Its Applications. Part I, Phys. Rep. 510, 87 (2012).

[5] R. Dean Astumian, Adiabatic Operation of a Molecular Machine, Proc. Natl. Acad. Sci. U.S.A. 104, 19715 (2007).

[6] N. A. Sinitsyn, The Stochastic Pump Effect and Geometric Phases in Dissipative and Stochastic Systems, J. Phys. A 42, 193001 (2009).

[7] R. Kubo, Statistical-Mechanical Theory of Irreversible Processes. I. General Theory and Simple Applications to Magnetic and Conduction Problems, J. Phys. Soc. Jpn. 12, 570 (1957).

[8] Joe Howard, Molecular Motors: Structural Adaptations to Cellular Functions, Nature (London) 389, 561 (1997).

[9] A. B. Kolomeisky and M. E. Fisher, Molecular Motors: A Theorist's Perspective, Annu. Rev. Phys. Chem. 58, 675 (2007).

[10] K. Visscher, M. J. Schnitzer, and S. M. Block, Single Kinesin Molecules Studied with a Molecular Force Clamp, Nature (London) 400, 184 (1999).

[11] M. E. Fisher and A. B. Kolomeisky, Simple Mechanochemistry Describes the Dynamics of Kinesin Molecules, Proc. Natl. Acad. Sci. U.S.A. 98, 7748 (2001). 
[12] R. Yasuda, H. Noji, K. Kinosita, and M. Yoshida, F1ATPase Is a Highly Efficient Molecular Motor that Rotates with Discrete 120 Degrees Steps, Cell 93, 1117 (1998).

[13] P. Gaspard and E. Gerritsma, The Stochastic Chemomechanics of the F1-ATPase Molecular Motor, J. Theor. Biol. 247, 672 (2007).

[14] W. R. Browne and B. L. Feringa, Making Molecular Machines Work, Nat. Nanotechnol. 1, 25 (2006).

[15] J. V. Hernández, E. R. Kay, and D. A. Leigh, A Reversible Synthetic Rotary Molecular Motor, Science 306, 1532 (2004).

[16] S. Erbas-Cakmak, D. A. Leigh, C. T. McTernan, and A. L. Nussbaumer, Artificial Molecular Machines, Chem. Rev. 115, 10081 (2015).

[17] D. A. Leigh, J. K. Y. Wong, F. Dehez, and F. Zerbetto, Unidirectional Rotation in a Mechanically Interlocked Molecular Rotor, Nature (London) 424, 174 (2003).

[18] N. A. Sinitsyn and I. Nemenman, The Berry Phase and the Pump Flux in Stochastic Chemical Kinetics, Europhys. Lett. 77, 58001 (2007).

[19] I. M. Sokolov, A Perturbation Approach to Transport in Discrete Ratchet Systems, J. Phys. A 32, 2541 (1999).

[20] Dibyendu Mandal and Christopher Jarzynski, A Proof by Graphical Construction of the No-Pumping Theorem of Stochastic Pumps, J. Stat. Mech. (2011) P10006.

[21] S. Rahav, J. Horowitz, and C. Jarzynski, Directed Flow in Nonadiabatic Stochastic Pumps, Phys. Rev. Lett. 101, 140602 (2008).

[22] V. Y. Chernyak and N. A. Sinitsyn, Pumping Restriction Theorem for Stochastic Networks, Phys. Rev. Lett. 101, 160601 (2008).

[23] C. Maes, K. Netočný, and S. R. Thomas, General No-Go Condition for Stochastic Pumping, J. Chem. Phys. 132, 234116 (2010).

[24] D. Mandal, Unification and New Extensions of the NoPumping Theorems of Stochastic Pumps, Europhys. Lett. 108, 50001 (2014).

[25] S. Asban and S. Rahav, No-Pumping Theorem for Many Particle Stochastic Pumps, Phys. Rev. Lett. 112, 050601 (2014).

[26] J. Ren, V. Y. Chernyak, and N. A. Sinitsyn, Duality and Fluctuation Relations for Statistics of Currents on Cyclic Graphs, J. Stat. Mech. (2011) P05011.

[27] S. Rahav, Extracting Work from Stochastic Pumps, J. Stat. Mech. (2011) P09020.

[28] M. Esposito and J. M. R. Parrondo, Stochastic Thermodynamics of Hidden Pumps, Phys. Rev. E 91, 052114 (2015).

[29] R. Uzdin, A. Levy, and R. Kosloff, Equivalence of Quantum Heat Machines, and Quantum-Thermodynamic Signatures, Phys. Rev. X 5, 031044 (2015).

[30] N. H. Lindner, G. Refael, and V. Galitski, Floquet Topological Insulator in Semiconductor Quantum Wells, Nat. Phys. 7, 490 (2011).

[31] K. I. Seetharam, C.-E. Bardyn, N. H. Lindner, M. S. Rudner, and G. Refael, Controlled Population of Floquet-Bloch States via Coupling to Bose and Fermi Baths, Phys. Rev. X 5, 041050 (2015).
[32] Y. H. Wang, H. Steinberg, P. Jarillo-Herrero, and N. Gedik, Observation of Floquet-Bloch States on the Surface of a Topological Insulator," Science 342, 453 (2013).

[33] M. C. Rechtsman, J. M. Zeuner, Y. Plotnik, Y. Lumer, D. Podolsky, F. Dreisow, S. Nolte, M. Segev, and A. Szameit, Photonic Floquet Topological Insulators, Nature (London) 496, 196 (2013).

[34] J. J. Hopfield, Kinetic Proofreading: A New Mechanism for Reducing Errors in Biosynthetic Processes Requiring High Specificity, Proc. Natl. Acad. Sci. U.S.A. 71, 4135 (1974).

[35] J. Ninio, Kinetic Amplification of Enzyme Discrimination, Biochimie 57, 587 (1975).

[36] J. Schnakenberg, Network Theory of Microscopic and Macroscopic Behavior of Master Equation Systems, Rev. Mod. Phys. 48, 571 (1976).

[37] U. Seifert, Stochastic Thermodynamics, Fluctuation Theorems and Molecular Machines, Rep. Prog. Phys. 75, 126001 (2012).

[38] R. K. P. Zia and B. Schmittmann, Probability Currents as Principal Characteristics in the Statistical Mechanics of Non-Equilibrium Steady States, J. Stat. Mech. (2007) $\mathrm{P} 07012$.

[39] If the coefficient matrix in these linear equations happens to be degenerate, then they may not have a unique solution. Generically, this degeneracy can be lifted by a small change in the choice of seed probabilities, $q_{j}^{a}$.

[40] D. Hartich, A. C. Barato, and U. Seifert, Nonequilibrium Sensing and Its Analogy to Kinetic Proofreading, New J. Phys. 17, 055026 (2015).

[41] A. Murugan, D. A. Huse, and S. Leibler, Speed, Dissipation, and Error in Kinetic Proofreading, Proc. Natl. Acad. Sci. U.S.A. 109, 12034 (2012).

[42] R. Rao and L. Peliti, Thermodynamics of Accuracy in Kinetic Proofreading: Dissipation and Efficiency TradeOffs, J. Stat. Mech. (2015) P06001.

[43] M. Tagliazucchi, E. A. Weiss, and I. Szleifer, Dissipative Self-Assembly of Particles Interacting through Time-Oscillatory Potentials, Proc. Natl. Acad. Sci. U.S.A. 111, 9751 (2014).

[44] K. Brandner, K. Saito, and U. Seifert, Thermodynamics of Micro- and Nano-Systems Driven by Periodic Temperature Variations, Phys. Rev. X 5, 031019 (2015).

[45] M. Esposito, R. Kawai, K. Lindenberg, and C. Van den Broeck, Efficiency at Maximum Power of LowDissipation Carnot Engines, Phys. Rev. Lett. 105, 150603 (2010).

[46] C. Van den Broeck, Thermodynamic Efficiency at Maximum Power, Phys. Rev. Lett. 95, 190602 (2005).

[47] M. Esposito, K. Lindenberg, and C. Van den Broeck, Universality of Efficiency at Maximum Power, Phys. Rev. Lett. 102, 130602 (2009).

[48] J. M. R. Parrondo, Reversible Ratchets as Brownian Particles in an Adiabatically Changing Periodic Potential, Phys. Rev. E 57, 7297 (1998).

[49] J. M. Horowitz and C. Jarzynski, Exact Formula for Currents in Strongly Pumped Diffusive Systems, J. Stat. Phys. 136, 917 (2009). 